\title{
Lamé polynomials, hyperelliptic reductions and Lamé band structure
}

\author{
By Robert S. MaIER \\ Depts. of Mathematics and Physics, University of Arizona, Tucson AZ 85721, \\ USA
}

The band structure of the Lamé equation, viewed as a one-dimensional Schrödinger equation with a periodic potential, is studied. At integer values of the degree parameter $\ell$, the dispersion relation is reduced to the $\ell=1$ dispersion relation, and a previously published $\ell=2$ dispersion relation is shown to be partially incorrect. The Hermite-Krichever Ansatz, which expresses Lamé equation solutions in terms of $\ell=1$ solutions, is the chief tool. It is based on a projection from a genus- $\ell$ hyperelliptic curve, which parametrizes solutions, to an elliptic curve. A general formula for this covering is conjectured, and is used to reduce certain hyperelliptic integrals to elliptic ones. Degeneracies between band edges, which can occur if the Lamé equation parameters take complex values, are investigated. If the Lamé equation is viewed as a differential equation on an elliptic curve, a formula is conjectured for the number of points in elliptic moduli space (elliptic curve parameter space) at which degeneracies occur. Tables of spectral polynomials and Lamé polynomials, i.e., band edge solutions, are given. A table in the older literature is corrected.

Keywords: Lamé equation, Lamé polynomial, dispersion relation, band structure, hyperelliptic reduction, Hermite-Krichever Ansatz

\section{Introduction}

\section{(a) Background}

The term 'Lamé equation' refers to any of several closely related second-order ordinary differential equations (Whittaker \& Watson 1927, Erdélyi 1953-55, Arscott 1964). The first such equation was obtained by Lamé, by applying the method of separation of variables to Laplace's equation in an ellipsoidal coordinate system. Lamé equations arise elsewhere in theoretical physics. Recent application areas include (i) the analysis of preheating after inflation, arising from parametric amplification (Boyanovsky et al. 1996, Greene et al. 1997, Kaiser 1998, Ivanov 2001); (ii) the stability analysis of critical droplets in bounded spatial domains (Maier \& Stein 2001); (iii) the stability analysis of static configurations in Josephson junctions (Caputo et al. 2000), (iv) the computation of the distance-redshift relation in inhomogeneous cosmologies (Kantowski \& Thomas 2001), and (v) magnetostatic problems in triaxial ellipsoids (Dobner \& Ritter 1998).

In some versions of the Lamé equation, elliptic functions appear explicitly, and in others (the algebraic versions) they appear implicitly. The version that appears 
most often in the physics literature is the Jacobi one,

$$
\left[-\frac{\mathrm{d}^{2}}{\mathrm{~d} \alpha^{2}}+\ell(\ell+1) m \operatorname{sn}^{2}(\alpha \mid m)\right] \Psi=E \Psi,
$$

which is a one-dimensional Schrödinger equation with a doubly periodic potential, parametrized by $m$ and $\ell$. Here $\operatorname{sn}(\cdot \mid m)$ is the Jacobi elliptic function with modular parameter $m . m$ is often restricted to $(0,1)$, though in general $m \in \mathbb{C} \backslash\{0,1\}$ is allowed. When $m \in(0,1)$, the function $\operatorname{sn}^{2}(\cdot \mid m)$ has real period $2 K \equiv 2 \mathrm{~K}(m)$ and imaginary period $2 \mathrm{i} K^{\prime} \equiv 2 \mathrm{i} K(1-m)$, with $\mathrm{K}(m)$ the first complete elliptic integral.

If $\alpha$ is restricted to the real axis and $m$ and $\ell$ are real, (1.1) becomes a realdomain Schrödinger equation with a periodic potential, i.e., a Hill's equation. Standard results on Hill's equation apply (Magnus \& Winkler 1979, McKean \& van Moerbeke 1979). Equipping (1.1) with a quasi-periodic boundary condition

$$
\Psi(\alpha+2 K)=\mathrm{e}^{\mathrm{i} k(2 K)} \Psi(\alpha) \equiv \xi \Psi(\alpha),
$$

where $k \in \mathbb{R}$ is fixed, defines a self-adjoint boundary value problem. For any real $k$ (i.e., for any Floquet multiplier $\xi$ with $|\xi|=1$ ), there will be an infinite discrete set of energies $E \in \mathbb{R}$ for which this problem has a solution, called a Bloch solution with crystal momentum $k$. Each such $E$ will lie in one of the allowed zones, which are intervals delimited by energies corresponding to $\xi= \pm 1$, i.e., to periodic and antiperiodic Bloch solutions. These form a sequence $E_{0}<E_{1} \leq E_{2}<E_{3} \leq E_{4}<\cdots$, where $E_{0}$ is a 'periodic' eigenvalue, followed by alternating pairs of anti-periodic and periodic eigenvalues (each pair may be coincident). The allowed zones are the intervals $\left[E_{2 j}, E_{2 j+1}\right]$. The complementary intervals $\left(E_{2 j+1}, E_{2 j+2}\right)$ are forbidden zones, or lacunæ. Any solution of Hill's equation with energy in a lacuna is unstable: its multiplier $\xi$ will not satisfy $|\xi|=1$, and its crystal momentum $k$ will not be real.

If the degree $\ell$ is an integer, which without loss of generality may be chosen to be non-negative, the Lamé equation (1.1) will have only a finite number of lacunæ. Its Bloch spectrum will consist of the $\ell+1$ bands $\left[E_{0}, E_{1}\right],\left[E_{2}, E_{3}\right], \ldots,\left[E_{2 \ell}, \infty\right)$. In this case, the potential $\ell(\ell+1) m \operatorname{sn}(\cdot \mid m)$ is said to be a finite-band or algebrogeometric potential. The $2 \ell+1$ band edges $E_{0}, \ldots, E_{2 \ell}$ will be algebraic functions of the parameter $m$. That is, the band edges will be the roots of a polynomial equation, the coefficients of which are polynomial in $m$. The corresponding periodic and antiperiodic Bloch solutions are called Lamé polynomials: they are polynomials in the Jacobi elliptic functions $\operatorname{sn}(\alpha \mid m), \operatorname{cn}(\alpha \mid m)$, and $\operatorname{dn}(\alpha \mid m)$. The double eigenvalues embedded in the topmost band $\left[E_{2 \ell}, \infty\right)$ (the 'conduction' band), namely $E_{2 j}=$ $E_{2 j+1}, j>\ell$, are loosely called transcendental eigenvalues. For $\ell=1$ at least, they are transcendental functions of $m$ (Chudnovksy \& Chudnovsky 1980).

There has been much work on algebraizing the integer- $\ell$ Lamé equation, to facilitate the computation of the band edges and the coefficients of the Lamé polynomials (Turbiner 1989, Li \& Kusnezov 1999, Li et al. 2000, Finkel et al. 2000). Such schemes have been extended to the case when $\ell$ is a half-odd-integer, in which there are an infinite number of lacunæ. In this case, certain 'mid-band' Bloch functions, namely ones with $\xi= \pm \mathrm{i}$ and real period $8 K$, are algebraic functions of $\operatorname{sn}(\alpha \mid m)$. Certain rational values of $\ell$ with $2 \ell \notin \mathbb{Z}$ also yield algebraic Bloch functions, provided the parameters $m$ and $E$ are chosen appropriately (Maier 2002).

An algebraic understanding of band edges is useful, but it is also desirable to have a closed-form expression for the dispersion relation: $k$ as a function of $E$. The 
value of $k$ is not unique, since it can be negated (equivalently, $\xi \mapsto 1 / \xi$ ), and any integer multiple of $\pi / K$ can be added. However, each branch has the property that $k \sim E^{1 / 2}$ or $k \sim-E^{1 / 2}$ to leading order as $E \rightarrow+\infty$. Also, $k \in \mathbb{R}$ in each band.

The goal of this paper is the efficient computation of the dispersion relation when $\ell$ is an integer. The following are illustrations of why this is of importance in theoretical physics. In application (i) above (preheating after inflation), particle production is due to parametric amplification: a solution having a multiplier $\xi$ with $|\xi|>1$. This corresponds to the energy $E$ not being at a band edge, or even in a band, but in a lacuna. In application (ii) (the stability analysis of a critical droplet), the analysis includes an imposition of Dirichlet rather than quasi-periodic boundary conditions on an $\ell=2$ Lamé equation (Maier \& Stein 2001). The resulting Bloch solution is not a Lamé polynomial, but rather a mid-band solution.

When $\ell$ is an integer, the Lamé equation is integrable, and the general integral of (1.1) can be expressed in terms of Jacobi theta functions. The dispersion relations $k=k_{\ell}(E \mid m), \ell \geq 1$, can in principle be computed in terms of elliptic integrals. The case $\ell=1$ is by far the easiest. If $\ell=1$, the solution space of (1.1), except when $E$ is at a band edge, will be spanned by the pair of Hermite-Halphen solutions

$$
\widetilde{\Phi}\left(\alpha ; \pm \alpha_{0} \mid m\right) \equiv \frac{\mathrm{H}\left(\alpha \mp \alpha_{0} \mid m\right)}{\Theta(\alpha \mid m)} \exp \left[\alpha \mathrm{Z}\left( \pm \alpha_{0} \mid m\right)\right] .
$$

Here $\mathrm{H}, \Theta, \mathrm{Z}$ are the Jacobi eta, theta and zeta functions, with periods $4 K, 2 K, 2 K$ respectively, and $\alpha_{0} \in \mathbb{C}$ is defined up to sign by $\operatorname{dn}^{2}\left(\alpha_{0} \mid m\right)=E-m$. So

$$
k_{1}(E \mid m)=-\mathrm{iZ}\left(\alpha_{0} \mid m\right)+\pi / 2 \mathrm{~K}(m), \quad \operatorname{dn}^{2}\left(\alpha_{0} \mid m\right)=E-m,
$$

up to multi-valuedness. This is a parametric dispersion relation. It has been exploited in a study of Wannier-Stark resonances with non-real $E$ and $k$ (Grecchi \& Sacchetti 1997, Sacchetti 1997). However, the extension to $\ell>1$ is numerically nontrivial. $k_{\ell}(E \mid m)$ turns out to equal $\sum_{r=0}^{\ell-1}\left[-\mathrm{iZ}\left(\alpha_{r} \mid m\right)+\pi / 2 \mathrm{~K}(m)\right]$, where $\left\{\alpha_{r}\right\}_{r=0}^{\ell-1}$ satisfy coupled transcendental equations involving $E$ and $m$ (Whittaker \& Watson $1927, \S 23.71)$. Li, Kusnezov and Iachello calculated and graphed $k=k_{2}(E \mid m)$ as well as $k=k_{1}(E \mid m)$ in the 'lemniscatic' case $m=\frac{1}{2}$ (Li \& Kusnezov 1999, Li et al. 2000). Unfortunately their graph of $k=k_{2}\left(E \mid \frac{1}{2}\right)$ is incorrect, as we shall show.

\section{(b) An overview}

When $\ell>1$, we abandon the traditional Hermite-Halphen solutions, and examine instead the implications for the Lamé dispersion relations of what is now called the Hermite-Krichever Ansatz. This is an alternative way of generating closed-form solutions of the Lamé solution at arbitrary energy $E$; for small integer values of $\ell$, at least. Until the 1980s, the only reference for the Ansatz was the classic work of Halphen (1888, chapitre XII), who applied it to the cases $\ell=2,3,4$, and in part to $\ell=5$. Krichever (1980) revived it as an aid in the construction of elliptic solutions of the Korteweg-de Vries and other integrable evolution equations. Belokolos et al. (1986) and Belokolos \& Enol'skii (2000) summarize early and recent developments.

The Hermite-Krichever Ansatz is easy to explain, even in the context of the Jacobi form of the Lamé equation, which is not the most convenient for symbolic manipulation. It asserts that for any integer $\ell \geq 1$, fundamental solutions of (1.1) 
can be constructed from the $\ell=1$ solutions $\widetilde{\Phi}\left(\alpha ; \pm \alpha_{0} \mid m\right)$ as finite series of the form

$$
\left[\sum_{j=0}^{N_{\ell}} C_{j}^{(\ell)} \frac{\mathrm{d}^{j}}{\mathrm{~d} \alpha^{j}} \widetilde{\Phi}\left(\alpha ; \pm \alpha_{0} \mid m\right)\right] \exp \left( \pm \kappa_{\ell} \alpha\right),
$$

where the parameter $\alpha_{0}$ is now computed from a reduced energy $\mathcal{E}_{\ell}$ by the formula $\mathrm{dn}^{2}\left(\alpha_{0} \mid m\right)=\mathcal{E}_{\ell}-m$. The reduced energy $\mathcal{E}_{\ell}$, the exponent $\kappa_{\ell}$, and the coefficients $\left\{C_{j}^{(\ell)}\right\}$ will depend on $E$ and $m . \mathcal{E}_{\ell}(E \mid m)$ may be chosen to be rational in $E$ and $m$, and $\pm \kappa_{\ell}$ to be of the form $\hat{\kappa}_{\ell}(E \mid m)$ times $\pm \mathrm{i} \sqrt{\widetilde{\mathrm{L}}_{\ell}(E \mid m)}$, where $\hat{\kappa}_{\ell}(E \mid m)$ is also rational, and $\widetilde{L}_{\ell}(E \mid m)$ is the spectral polynomial $\prod_{s=0}^{2 \ell}\left[E-E_{s}(m)\right]$, a degree- $(2 \ell+1)$ polynomial in $E$ the coefficients of which, as noted, are rational in $m$.

If the Lamé equation can be integrated in the framework of the HermiteKrichever Ansatz, it follows from (1.5) that that up to sign, etc.,

$$
k_{\ell}(E \mid m)=k_{1}\left(\mathcal{E}_{\ell}(E \mid m) \mid m\right)+\hat{\kappa}_{\ell}(E \mid m) \sqrt{\widetilde{\mathrm{L}}_{\ell}(E \mid m)} .
$$

The dispersion relation for any integer $\ell$ can be expressed in terms of the $\ell=1$ relation. To compute $k_{\ell}$, only one transcendental function (i.e., $k_{1}$ ) needs to be evaluated, since the other functions in (1.6) are elementary. The only difficult matter is choosing the relative sign of the two terms, since each is defined only up to sign.

The functions $\mathcal{E}_{\ell}(E \mid m), \hat{\kappa}_{\ell}(E \mid m)$ are rational with integer coefficients, but working them out when $\ell$ is large is a lengthy task. In principle one can write down a recurrence relation for the coefficients $\left\{C_{j}\right\}$, and work out $\mathcal{E}_{\ell}(E \mid m), \hat{\kappa}_{\ell}(E \mid m)$ from the condition that the series terminate. However, their numerator and denominator degrees grow quadratically as $\ell$ increases. This explains why Halphen's treatment of the $\ell=5$ case was only partial. In a series of papers, Kostov, Enol'skii, and collaborators used computer algebra systems to perform a full analysis of the cases $\ell=2,3,4,5$ (Gerdt \& Kostov 1989, Kostov \& Enol'skii 1993, Enol'skii \& Kostov 1994, Eilbeck \& Enol'skii 1994). When $\ell=5$, using Mathematica to compute the integer coefficients of rational functions equivalent to $\mathcal{E}_{\ell}(E \mid m), \hat{\kappa}_{\ell}(E \mid m)$ required seven hours of time on a Sparc-1, a Unix workstation of that era (Eilbeck \& Enol'skii 1994). Until now, their analysis has not been extended to higher $\ell$.

On the basis of extensive symbolic computation, we make a conjecture that we call Conjecture L. For all integer $\ell \geq 2$, the degree- $\ell$ Lamé equation can be integrated in the framework of the Hermite-Krichever Ansatz, and the rational functions that perform the reduction to the $\ell=1$ case can be computed, by simple formulas that we supply, from certain spectral polynomials of the degree- $\ell$ equation, which are relatively easy to work out. These are the standard spectral polynomial $\prod_{s=0}^{2 \ell}\left[E-E_{s}(m)\right]$ associated with the band-edge solutions, and the spectral polynomials associated with two other types of closed-form solution that have not previously been studied in the literature. We call them twisted and theta-twisted Lamé polynomials. In the context of the Jacobi form, the former are polynomials in $\operatorname{sn}(\alpha \mid m), \operatorname{cn}(\alpha \mid m)$ and $\operatorname{dn}(\alpha \mid m)$, multiplied by a factor $\exp (\kappa \alpha)$. (If $\kappa \in \mathbb{R}$, 'canted' would be better than 'twisted'.) The latter contain a factor resembling (1.3).

Conjecture $\mathrm{L}$, which we have verified for all $\ell \leq 8$, simplifies the computation of the dispersion relations. It also has implications for hyperelliptic reduction: the reduction of hyperelliptic integrals to elliptic ones (Belokolos et al. 1986). This is on 
account of the following. For any $\ell \geq 1$ and $m$, the solutions of the Lamé equation, both the Hermite-Halphen solutions and those derived from the Hermite-Krichever Ansatz, are single-valued functions not of $E$, but rather of a point $(E, \tilde{\nu})$ on the $\ell$ 'th Lamé spectral curve: a hyperelliptic curve comprising all $(E, \tilde{\nu})$ satisfying

$$
\tilde{\nu}^{2}=\prod_{s=0}^{2 \ell}\left[E-E_{s}(m)\right] .
$$

The fact that $\tilde{\nu}=\tilde{\nu}(E)$ is two-valued (except at a band edge) is responsible for the two-valuedness of, e.g., the parameter $\alpha_{0}=\alpha_{0}(E)$ of (1.3), and in general, for the uncertainty in the sign of $k$. The $\ell$ 'th spectral curve generically has genus $\ell$ and may be denoted $\widetilde{\Gamma}_{\ell} \equiv \widetilde{\Gamma}_{\ell}(m)$. For any integer $\ell \geq 2$, the $E \mapsto \mathcal{E}_{\ell}(E \mid m)$ reduction map of the Hermite-Krichever Ansatz induces a covering $\pi_{\ell}: \widetilde{\Gamma}_{\ell} \rightarrow \widetilde{\Gamma}_{1}$. The first known covering of an elliptic curve by a higher-genus hyperelliptic curve was constructed by Legendre and generalized by Jacobi (Belokolos et al. 1986, §2). But it is difficult to enumerate such coverings, or even work out explicit examples. Those generated by the Ansatz applied to the Lamé equation are a welcome exception.

The integral with respect to $E$ of any rational function of $E$ and $\sqrt{\Pi(E)}$, where $\Pi$ is a polynomial, is a line integral on the algebraic curve defined by $\tilde{\nu}^{2}=\Pi(E)$. The covering $\pi_{\ell}: \widetilde{\Gamma}_{\ell} \rightarrow \widetilde{\Gamma}_{1}$, a formula for which is provided by Conjecture L, reduces certain such hyperelliptic integrals to elliptic ones. In modern language, the conjecture specifies how certain holomorphic differentials on hyperelliptic curves can arise as pullbacks of holomorphic differentials on elliptic curves.

We investigate the degeneracies of the band edges $\left\{E_{s}(m)\right\}_{s=0}^{2 \ell}$ that can occur if the modular parameter $m$ is non-real. The existence of such level-crossings was pointed out by Cohn (1888), in a dissertation that seems not to have been followed up, though it was later cited by Whittaker \& Watson $(1927, \S 23.41)$. We conjecture a formula for the $\ell$-dependence of the number of values of $m \in \mathbb{C} \backslash\{0,1\}$, or equivalently the number of values of the Klein invariant $J \in \mathbb{C}$, at which two band edges coincide. When this occurs, the genus of the hyperelliptic spectral curve $\widetilde{\Gamma}_{\ell}$ is reduced from $\ell$ to $\ell-1$. Band-edge degeneracies are responsible for a fact discovered by Turbiner (1989): if $\ell \geq 2$, the complex curve comprising all points $\left\{\left(m, E_{s}(m)\right)\right\}_{s=0}^{2 \ell}$ in $\mathbb{C} \backslash\{0,1\} \times \mathbb{C}$ has only four, rather than $2 \ell+1$, connected components.

This paper is organized as follows. Section 2 introduces an alternative form of the Lamé equation, and Lamé polynomials in the context of the new form are classified in $\S 3$. The Hermite-Krichever Ansatz and Conjecture L are introduced in $\S 4$. The application to hyperelliptic reduction is covered in $\S 5$. In $\S 6-\S 7$, dispersion relations are worked out and the abovementioned dispersion relation for the case $\ell=2$ is corrected. The dispersion relation for $\ell=3$ is graphed as well. Finally, an area for future investigation, besides Conjecture L itself, is mentioned in $\S 8$.

\section{The elliptic-curve algebraic form}

In $\S 3-\S 6$ we use exclusively what we call the elliptic-curve algebraic form of the Lamé equation, which is the most convenient for symbolic computation. In this section we derive it, and define a fundamental multi-valued function $\Phi$ (nowadays called an elliptic Baker-Akhiezer function) which appears in the elliptic-curve version of both the Hermite-Halphen solutions and the Hermite-Krichever Ansatz. 
Many algebraic forms can be obtained from (1.1) by changing to new independent variables which are elliptic functions of $\alpha$, such as $\operatorname{sn}(\alpha \mid m)$. (See, e.g., Arscott \& Khabaza (1962, §1.1) and Arscott (1964, pp 192-3)). A form in which the domain of definition is explicitly a cubic algebraic curve of genus 1, i.e., a cubic elliptic curve, can be obtained as follows. First, the Lamé equation is restated in terms of the Weierstrassian function $\wp=\wp\left(u ; g_{2}, g_{3}\right)$. This is the canonical elliptic function with a double pole at $u=0$, satisfying $\left(\wp^{\prime}\right)^{2}=f(\wp)$ where $f(x) \equiv 4 x^{3}-g_{2} x-g_{3}=4 \prod_{\gamma=1}^{3}\left(x-e_{\gamma}\right)$. For ellipticity the roots $\left\{e_{\gamma}\right\}_{\gamma=1}^{3}$ must be distinct, which is equivalent to the condition that the modular discriminant $\Delta \equiv g_{2}^{3}-27 g_{3}^{2}$ be non-zero. Either of $g_{2}, g_{3} \in \mathbb{C}$ may equal zero, but not both.

The relation between the Jacobi and Weierstrass elliptic functions is well-known (Abramowitz \& Stegun 1965, §18.9). Choose $\left\{e_{\gamma}\right\}_{\gamma=1}^{3}$ according to

$$
\left(e_{1}, e_{2}, e_{3}\right)=A^{2}\left(\frac{2-m}{3}, \frac{2 m-1}{3}, \frac{-(m+1)}{3}\right),
$$

where $A \in \mathbb{C} \backslash\{0\}$ is any convenient proportionality constant. Then

$$
g_{2}=A^{4} \frac{4\left(m^{2}-m+1\right)}{3}, \quad g_{3}=A^{6} \frac{4(m-2)(2 m-1)(m+1)}{27},
$$

and the dimensionless ( $A$-independent) Klein invariant $J \equiv g_{2}^{3} / \Delta$ will be given by

$$
J=\frac{4}{27} \frac{\left(m^{2}-m+1\right)^{3}}{m^{2}(1-m)^{2}} .
$$

The two sorts of elliptic function will be related by, e.g.,

$$
\mathrm{sn}^{2}(A z \mid m)=\frac{e_{1}-e_{3}}{\wp(z)-e_{3}}, \quad \mathrm{~ns}^{2}(A z \mid m)=\frac{\wp(z)-e_{3}}{e_{1}-e_{3}}
$$

and the periods of $\wp$, denoted $2 \omega, 2 \omega^{\prime}$, will be related to those of $\operatorname{sn}^{2}$ by

$$
2 \omega=2 K / A, \quad 2 \omega^{\prime}=2 \mathrm{i} K^{\prime} / A .
$$

The case when $2 K, 2 K^{\prime}$ are real, or equivalently $\omega \in \mathbb{R}, \omega^{\prime} \in \mathrm{i} \mathbb{R}$ (we assume $A \in \mathbb{R}$ ), is the case when $g_{2}, g_{3} \in \mathbb{R}$ and $\Delta>0$ (Abramowitz \& Stegun 1965, §18.1).

Choosing for simplicity $A=1$, so that $e_{1}-e_{3}=A^{2}=1$, and rewriting the Lamé equation (1.1) with the aid of (2.4), yields the Weierstrassian form

$$
\left\{\frac{\mathrm{d}^{2}}{\mathrm{~d} u^{2}}-\left[\ell(\ell+1) \wp\left(u ; g_{2}, g_{3}\right)+B\right]\right\} \Psi=0,
$$

where $u \equiv \alpha+\mathrm{i} K^{\prime}$. (The translation of (1.1) by i $K^{\prime}$ replaces $m \mathrm{sn}^{2}$ by $\mathrm{ns}^{2}$.) Here $B \equiv-E\left(e_{1}-e_{3}\right)-\ell(\ell+1) e_{3}$, i.e.,

$$
B \equiv-E+\frac{1}{3} \ell(\ell+1)(m+1),
$$

is a transformed energy parameter. Changing to the new independent variable $x \equiv$ $\wp\left(u ; g_{2}, g_{3}\right)$ converts $(2.6)$ to the commonly encountered algebraic form

$$
\left\{\frac{\mathrm{d}^{2}}{\mathrm{~d} x^{2}}+\frac{1}{2} \sum_{\gamma=1}^{3} \frac{1}{x-e_{\gamma}} \frac{\mathrm{d}}{\mathrm{d} x}-\frac{\ell(\ell+1) x+B}{4 \prod_{\gamma=1}^{3}\left(x-e_{\gamma}\right)}\right\} \Psi=0 .
$$


This is a differential equation on the Riemann sphere $\mathbb{P}^{1} \equiv \mathbb{C} \cup\{\infty\}$ with regular singular points at $x=e_{1}, e_{2}, e_{3}, \infty$. Any solution of the original Lamé equation (1.1) or the Weierstrassian form (2.6) which is quasi-periodic in the sense that it is multiplied by $\xi, \xi^{\prime} \in \mathbb{C} \backslash\{0\}$ when $\alpha \leftarrow \alpha+2 K$ or $\alpha \leftarrow \alpha+2 \mathrm{i} K^{\prime}$ respectively (equivalently, when $u \leftarrow u+2 \omega$ or $u \leftarrow u+2 \omega^{\prime}$ ), will be a path-multiplicative function of $x$. That is, it will be multiplied by $\xi, \xi^{\prime}$ when it is analytically continued around a cut joining the pair of points $x=e_{2}, e_{3}$ or $x=e_{1}, e_{2}$, respectively. In the context of the algebraic form, the dispersion relation is still a relation between the energy and a multiplier $\xi$, but the multiplier is interpreted as specifying not quasi-periodicity on $\mathbb{C}$, but rather multi-valuedness on $\mathbb{P}^{1}$.

The algebraic form (2.8) of the Lamé equation lifts naturally to the complex elliptic curve $E_{g_{2}, g_{3}} \equiv\left\{(x, y) \in \mathbb{C}^{2} \mid y^{2}=f(x)\right\} \cup\{(\infty, \infty)\}$ over $\mathbb{P}^{1}$, parametrized by $(x, y) \equiv\left(\wp(u), \wp^{\prime}(u)\right)$. One can rewrite (2.8) in the elliptic-curve algebraic form

$$
\left\{\left(y \frac{\mathrm{d}}{\mathrm{d} x}\right)^{2}-[\ell(\ell+1) x+B]\right\} \Psi=0,
$$

which also follows from (2.6), since $\mathrm{d} / \mathrm{d} u=\wp^{\prime} \mathrm{d} / \mathrm{d} \wp=y \mathrm{~d} / \mathrm{d} x$. This is a differential equation on $E_{g_{2}, g_{3}}$ with a single singular point: a regular one at $(x, y)=(\infty, \infty)$. Note that the 2-to- 1 covering map $\pi: E_{g_{2}, g_{3}} \rightarrow \mathbb{P}^{1}$ defined by $\pi(x, y)=x$ has $\left\{\left(e_{\gamma}, 0\right)\right\}_{\gamma=1}^{3}$ and $(\infty, \infty)$ as simple critical points. One reason why $(2.9)$ is more fundamental than (2.8) is that the singular points of (2.8) at $x=e_{1}, e_{2}, e_{3}$ can be regarded as artifacts: consequences of $\left\{\left(e_{\gamma}, 0\right)\right\}_{\gamma=1}^{3}$ being critical points of $\pi$.

The complex-analytic differential geometry of the elliptic curve $E_{g_{2}, g_{3}}$ takes a bit of getting used to. Both $x$ and $y$ are meromorphic $\mathbb{P}^{1}$-valued functions on $E_{g_{2}, g_{3}}$, and the only pole that either has on $E_{g_{2}, g_{3}}$ is at the point $O \equiv(\infty, \infty)$. In a neighborhood of any point $(x, y)$ other than $O$ and the three points $\left(e_{\gamma}, 0\right)$, either $x$ or $y$ will serve as a local coordinate. However, near each $\left(e_{\gamma}, 0\right)$ only $y$ will be a good local coordinate, since $\mathrm{d} y / \mathrm{d} x$ diverges at $x=e_{\gamma}$. Also, $x$ has a double and $y$ has a triple pole at $O$, so the appropriate local coordinate near $O$ is the quotient $x / y$. The 1 -form $\mathrm{d} x / y$ is not merely meromorphic but holomorphic, with no poles on $E_{g_{2}, g_{3}}$. Its dual is the vector field (or directional derivative) $y \mathrm{~d} / \mathrm{d} x$.

Elliptic functions, i.e., doubly periodic functions, of the original variable $u \in \mathbb{C}$ correspond to single-valued functions on $E_{g_{2}, g_{3}}$. These are rational functions of $x, y$ and may be written as $R_{0}(x)+R_{1}(x) y$, i.e., $R_{0}(\wp(u))+R_{1}(\wp(u)) \wp^{\prime}(u)$. The formula $(y \mathrm{~d} / \mathrm{d} x) y=6 x^{2}-\frac{1}{2} g_{2}$ allows such functions to be differentiated algebraically. In a similar way, quasi-doubly periodic functions of $u$ (sometimes called elliptic functions of the second kind), which are multiplied by $\xi$ when $u \leftarrow u+2 \omega$ and by $\xi^{\prime}$ when $u \leftarrow u+2 \omega^{\prime}$, correspond to multiplicatively multi-valued functions on $E_{g_{2}, g_{3}}$.

$E_{g_{2}, g_{3}}$ has genus 1 and is topologically a torus. A fundamental pair of loops that cannot be shrunk to a point may be chosen to be a loop that extends between $\left(e_{2}, 0\right)$ and $\left(e_{3}, 0\right)$, and one that extends between $\left(e_{1}, 0\right)$ and $\left(e_{2}, 0\right)$, with (if $g_{2}, g_{3}$ are real, at least) half of each loop passing through positive values of $y$, and the other half through negative values. One way of constructing an elliptic function of the second kind is to anti-differentiate a rational function $R(x, y)$. Here 'antidifferentiate' means to compute $\int R(x, y) \mathrm{d} x / y$, its indefinite integral against the holomorphic 1-form $\mathrm{d} x / y$. The resulting function will typically have a non-zero 
Table 1. Hermite-Halphen polynomials (van der Waall 2002, table A.2).

\begin{tabular}{ll}
\hline$\ell$ & $\hat{\mathcal{F}}_{\ell}\left(x ; B ; g_{2}, g_{3}\right)$ \\
\hline 1 & $x-B$ \\
2 & $x^{2}-\frac{1}{3} B x+\left(\frac{1}{9} B^{2}-\frac{1}{4} g_{2}\right)$ \\
3 & $x^{3}-\frac{1}{5} B x^{2}+\left(\frac{2}{75} B^{2}-\frac{1}{4} g_{2}\right) x+\left(-\frac{1}{225} B^{3}+\frac{1}{15} B g_{2}-\frac{1}{4} g_{3}\right)$ \\
\hline
\end{tabular}

modulus of periodicity associated with each loop. If so, exponentiating it will yield a multiplicatively multi-valued function on $E_{g_{2}, g_{3}}$, with non-unit multipliers $\xi, \xi^{\prime}$.

To illustrate this, we briefly sketch the construction of the Hermite-Halphen solutions of the elliptic-curve algebraic Lamé equation (2.9), for integer $\ell \geq 1$. This exposition is a bit novel, since the standard published exposition is not fully algebraic, being largely framed in the context of the Weierstrassian form (Whittaker \& Watson 1927, §23.7). The starting point is the differential equation on $E_{g_{2}, g_{3}}$

$$
\left\{\left(y \frac{\mathrm{d}}{\mathrm{d} x}\right)^{3}-4[\ell(\ell+1) x+B]\left(y \frac{\mathrm{d}}{\mathrm{d} x}\right)-2 \ell(\ell+1) y\right\} \mathcal{F}=0 .
$$

The differential operator in (2.10) is the 'symmetric square' of the Lamé operator of (2.9), so the solutions of (2.10) include the product of any pair of solutions of (2.9). By examination, the solutions of (2.10) include a polynomial in $x$ (and also in $\left.B, g_{2}, g_{3}\right)$, of degree $\ell$ in both $x$ and $B$. We write it $\hat{\mathcal{F}}_{\ell}$ or $\mathcal{F}_{\ell}$ when it is normalized to be monic in $x, B$ respectively. It can be shown that $\mathcal{F}_{\ell}=(-1)^{\ell}[(2 \ell-1) ! !] \hat{\mathcal{F}}_{\ell}$. These are so-called Hermite-Halphen polynomials (see table 1).

It follows from manipulations parallel to those of Whittaker \& Watson that the functions $\Psi_{\ell}^{ \pm}$on $E_{g_{2}, g_{3}}$ defined by a formula containing an indefinite integral,

$$
\Psi_{\ell}^{ \pm}\left(x, y ; B ; g_{2}, g_{3}\right) \equiv \exp \int\left[\frac{\frac{1}{2} \mathcal{F}_{\ell}^{\prime}\left(x ; B ; g_{2}, g_{3}\right) y \pm \nu^{(\ell)}\left(B ; g_{2}, g_{3}\right)}{\mathcal{F}_{\ell}\left(x ; B ; g_{2}, g_{3}\right)}\right] \frac{\mathrm{d} x}{y},
$$

are solutions of the Lamé equation (2.9). Here $\nu^{(\ell)}$ is an energy-dependent but position-independent quantity that can be computed from the Hermite-Halphen polynomial by what Whittaker \& Watson term an 'interesting formula',

$$
\nu^{(\ell)^{2}}=-\frac{1}{2} \mathcal{F}_{\ell}\left(y \frac{\mathrm{d}}{\mathrm{d} x}\right) \mathcal{F}_{\ell}+\left[\frac{1}{2}\left(y \frac{\mathrm{d}}{\mathrm{d} x}\right) \mathcal{F}_{\ell}\right]^{2}+[\ell(\ell+1) x+B] \mathcal{F}_{\ell}^{2}
$$

(It is not obvious that the right-hand side is independent of the point $(x, y) \in$ $E_{g_{2}, g_{3}}$.) It is widely known (Smirnov 2002) that $\nu^{(\ell)}$ is identical to the coordinate $\nu$ on the spectral curve defined by $\nu^{2}=\prod_{s=0}^{2 \ell}\left[B-B_{s}\left(g_{2}, g_{3}\right)\right]$, which will be studied in $\S 4$ and $\S 5$; though no simple proof of this fact seems to have been published. The Hermite-Halphen polynomials are algebraically interesting in their own right. Klein (1892, figures 1,2) provides a sketch of the real portion of the curve $\mathcal{F}_{\ell}(x ; B)=0$ when $\ell=5,6$, showing how when $\ell \geq 4, B$ is a band edge only if $\mathcal{F}_{\ell}(x ; B)$, regarded as a polynomial in $x$, has a double root.

The functions $\Psi_{\ell}^{ \pm}$defined by (2.11) are the Hermite-Halphen solutions of the degree- $\ell$ Lamé equation in its elliptic-curve algebraic form. If $\nu^{(\ell)}\left(B ; g_{2}, g_{3}\right) \neq 0$, they are distinct. They are path-multiplicative as promised, since they are exponentials of anti-derivatives of rational functions on $E_{g_{2}, g_{3}}$. 
Definition. On the elliptic curve $E_{g_{2}, g_{3}}$, the multi-valued meromorphic function $\Phi$, parametrized by $\left(x_{0}, y_{0}\right) \in E_{g_{2}, g_{3}} \backslash\{(\infty, \infty)\}$, is defined up to a constant factor by a formula containing an indefinite elliptic integral,

$$
\Phi\left(x, y ; x_{0}, y_{0}\right)=\exp \left[\frac{1}{2} \int\left(\frac{y+y_{0}}{x-x_{0}}\right) \frac{\mathrm{d} x}{y}\right] .
$$

Its multi-valuedness, which is multiplicative, arises from the path of integration winding around $E_{g_{2}, g_{3}}$ in any combination of the two directions. Each branch of $\Phi$ has a simple zero at $(x, y)=\left(x_{0}, y_{0}\right)$ and a simple pole at $(x, y)=(\infty, \infty)$.

This multi-valued function $\Phi$ is an algebraic version of Halphen's l'élément simple (Halphen 1888), now called an elliptic Baker-Akhiezer function (Krichever 1990). It follows from (2.11), and the fact that $\mathcal{F}_{1}$ equals $B-x$ (see table 1 ), that

$$
\Psi_{1}^{ \pm}\left(x, y ; B ; g_{2}, g_{3}\right)=\Phi\left(x, y ; B, \pm \sqrt{4 B^{3}-g_{2} B-g_{3}}\right) .
$$

That is, if $\left(x_{0}, y_{0}\right) \in E_{g_{2}, g_{3}}$ is 'above' $x_{0}=B$, then $\Phi\left(\cdot, \cdot ; x_{0}, y_{0}\right)$ will be a solution of the $\ell=1$ Lamé equation in the form (2.9). There are two such points, related by $y_{0}$ being negated, unless $4 B^{3}-g_{2} B-g_{3}=0$, i.e., unless $B=e_{1}, e_{2}, e_{3}$, in which case $y_{0}=0$ is the only possibility. These are the three band-edge values of $B$ for $\ell=1$.

It is not difficult to show that the $\ell=1$ Hermite-Halphen solutions (2.14) are identical to the solutions (1.3), though they are expressed as functions of the variable $(x, y) \in E_{g_{2}, g_{3}}$ rather than the original independent variable $\alpha \in \mathbb{C}$. The parametrizing point $\left(x_{0}, \pm y_{0}\right) \in E_{g_{2}, g_{3}}$ corresponds to the parameter $\pm \alpha_{0} \in \mathbb{C}$ of (1.3). These solutions are clearly easier to formulate in the elliptic curve context.

For any integer $\ell$, the Lamé dispersion relation can be computed numerically from (2.11) by calculating the multiplier arising from the path of integration winding around $E_{g_{2}, g_{3}}$. However, (2.11) is not adapted to symbolic computation. By expanding the integrand in partial fractions one can derive the remarkable formula

$$
\Psi_{\ell}^{ \pm}\left(x, y ; B ; g_{2}, g_{3}\right)=\prod_{r=1}^{\ell} \Phi\left(x, y ; x_{r}, y_{r}^{ \pm}\right),
$$

where $\left\{\left(x_{r}, y_{r}^{ \pm}\right)\right\}_{r=1}^{\ell}$ are points on $E_{g_{2}, g_{3}}$ above $\left\{x_{r}\right\}_{r=1}^{\ell}$, the $B$-dependent roots of the degree- $\ell$ polynomial $\mathcal{F}_{\ell}\left(x ; B ; g_{2}, g_{3}\right)$. (Cf. Whittaker \& Watson $(1927, \S 23.7)$.) Unfortunately, when $\ell \geq 5$ the roots $\left\{x_{r}\right\}_{r=1}^{\ell}$ cannot be computed in terms of radicals. This reduction to degree- 1 solutions is less computationally tractable than the one that will be provided by the Hermite-Krichever Ansatz.

\section{Finite families of Lamé equation solutions}

The solutions of the integer- $\ell$ Lamé equation include the Lamé polynomials, which are the traditional band-edge solutions. In the Jacobi-form context they are periodic or anti-periodic functions on $[0,2 K]$, with Floquet multiplier $\xi= \pm 1$, respectively. There are exactly $2 \ell+1$ values of the parameter $B \in \mathbb{C}$, i.e., of the energy $E$, for which a Lamé polynomial may be constructed, the counting being up to multiplicity. By definition these are the roots of the spectral polynomial $\mathrm{L}_{\ell}\left(B ; g_{2}, g_{3}\right)$.

As functions on the curve $E_{g_{2}, g_{3}}$, the Lamé polynomials are single or doublevalued and are essentially polynomials in the coordinates $x, y$. (In the Weierstrassian 
context $\wp, \wp^{\prime}$ substitute for $x, y$.) However, no fully satisfactory table of the Lamé polynomials or the spectral polynomials has yet been published. Whittaker \& Watson $(1927, \S 23.42)$ refer to a list of Guerritore (1909) that covers $\ell \leq 10$. Sadly, although he produced it as a dissertazione di laurea at the University of Naples, most of his results on $\ell \geq 5$ are incorrect. This has long been known (Strutt 1967), but his paper is still occasionally cited (Gesztesy \& Holden 2003). Arscott (1964, §9.3.2) gives a brief table of the Jacobi-form Lamé polynomials, covering only $\ell=1,2,3$. His table is correct, with a single misprint (Fernández C. et al. 2000, Finkel et al. 2000). But its brevity has been misinterpreted. An erroneous belief has arisen that when $\ell \geq 4$, the Lamé polynomial coefficients and band edge energies cannot be expressed in terms of radicals. This actually sets in only when $\ell \geq 8$.

Due to these confusions, in this section we tabulate the Lamé polynomials and the spectral polynomials $\mathrm{L}_{\ell}\left(B ; g_{2}, g_{3}\right)$. Both are computed from coefficient recurrence relations. We supply such relations and tables of spectral polynomials for the twisted and theta-twisted Lamé polynomials, as well. The number of values of $B \in \mathbb{C}$ for which the latter two sorts of solution exist, i.e., the degrees of their spectral polynomials, are not known, but in each case we conjecture a formula. All three sorts of solution will play a role in Conjecture L. In fact, all will be special cases of the solutions constructed for arbitrary $B$ by the Hermite-Krichever Ansatz.

When $\ell \geq 2$, many of the spectral polynomials will have degenerate roots if $g_{2}, g_{3} \in \mathbb{C}$ are appropriately chosen. This means that, for example, a pair of the $2 \ell+1$ band edge energies can be made to coincide by moving the modular parameter $m \in$ $\mathbb{C} \backslash\{0,1\}$ to one of a set of complex values. We indicate how to calculate these values, or the corresponding values of Klein's absolute invariant $J=g_{2}^{3} /\left(g_{2}^{3}-27 g_{3}^{2}\right) \in \mathbb{C}$.

$J$ is the more fundamental parameter, in algebraic geometry at least, since two elliptic curves are isomorphic (birationally equivalent) if and only if they have the same value of $J$. The $m \mapsto J$ correspondence (2.3) maps $\mathbb{C} \backslash\{0,1\}$ onto $\mathbb{C}$, and it also maps $m \in \mathbb{R} \backslash\{0,1\}$ (in fact, $m \in\left(0, \frac{1}{2}\right]$ ) onto $J \in[1, \infty)$ ). Formally it is 6 -to-1. Each value of $J$ corresponds to six values of $m$, with the exception of $J=0$ (i.e., $g_{2}=0$ ), which corresponds to $m=\frac{1}{2} \pm \frac{\sqrt{3}}{2}$ i, and $J=1$ (i.e., $g_{3}=0$ ), which corresponds to $m=-1, \frac{1}{2}, 2$. Elliptic curves with $J=0,1$ are called equianharmonic and lemniscatic, respectively (Abramowitz \& Stegun 1965, §18.13-§18.15). Any equianharmonic curve has a triangular period lattice, with $\omega^{\prime} / \omega=\mathrm{e}^{ \pm 2 \pi \mathrm{i} / 3}$, and any lemniscatic curve has a square period lattice, with $\omega^{\prime} / \omega= \pm$ i.

\section{(a) Lamé polynomials}

The Lamé polynomials are classified into species 1,2,3,4 (Whittaker \& Watson $1927, \S 23.2)$. This is appropriate for some forms of the Lamé equation, but for the elliptic-curve algebraic form, a more structured classification scheme is better.

Definition. A solution of the Lamé equation (2.9) on the elliptic curve $E_{g_{2}, g_{3}}$ is said to be a Lamé polynomial of Type $\mathrm{I}$ if it is single-valued and of the form $C(x)$ or $D(x) y$, where $C, D$ are polynomials. A solution is said to be a Lamé polynomial of Type II, associated with the branch point $e_{\gamma}$ of the curve $(\gamma=1,2,3)$, if it is double-valued and of the form $E(x) \sqrt{x-e_{\gamma}}$ or $F(x) y / \sqrt{x-e_{\gamma}}$, where $E, F$ are polynomials. The subtypes of Types I and II are species 1, 4 and 2,3, respectively. 
Table 2. Lamé polynomial solutions of Types I,II

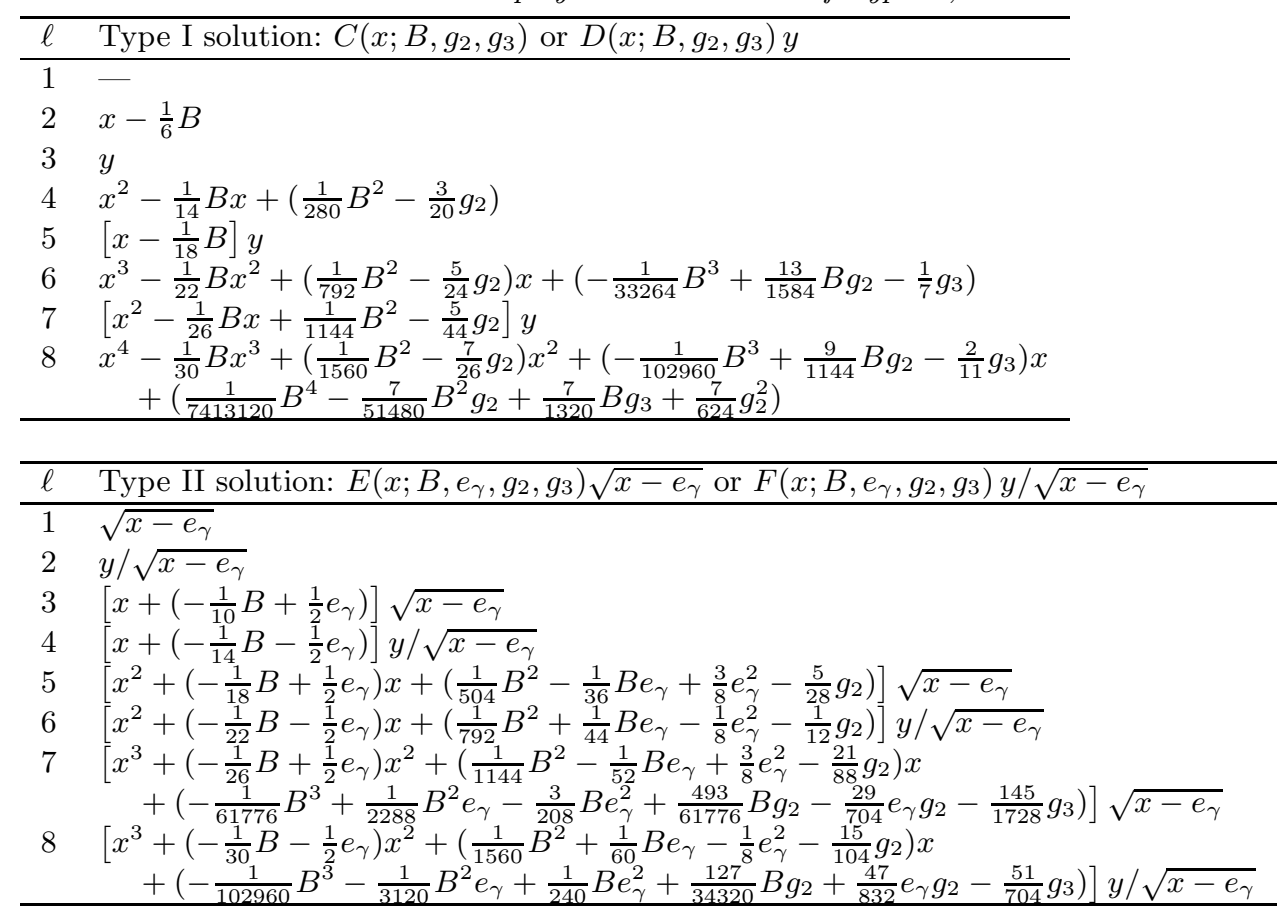

To determine necessary conditions on $\ell$ and $B$ for there to be a nonzero Lamé polynomial of each subtype, we substitute the corresponding expression $(C(x)$, etc.) into the Lamé equation (2.9), and work out a recurrence for the polynomial coefficients. This is similar to the approach of expanding in integer or half-integer powers of $x-e_{\gamma}$ (Whittaker \& Watson 1927, §23.41), though it leads to four-term rather than three-term recurrences. For the Type I solutions at least, the present approach seems more natural, since they are not associated with any branch point $e_{\gamma}$.

If $C(x)=\sum_{j} c_{j} x^{j}, D(x)=\sum_{j} d_{j} x^{j}, E(x)=\sum_{j} e_{j} x^{j}$, and $F(x)=\sum_{j} f_{j} x^{j}$, substituting the expression for each species of solution into (2.9) and equating the coefficients of powers of $x$ leads to the recurrence relations

$$
\begin{aligned}
& (2 j-\ell)(2 j+\ell+1) c_{j}-B c_{j+1} \\
& \quad-(j+2)\left(j+\frac{3}{2}\right) g_{2} c_{j+2}-(j+2)(j+3) g_{3} c_{j+3}=0 \\
& (2 j-\ell+3)(2 j+\ell+4) d_{j}-B d_{j+1} \\
& \quad-(j+2)\left(j+\frac{5}{2}\right) g_{2} d_{j+2}-(j+2)(j+3) g_{3} d_{j+3}=0 \\
& (2 j-\ell+1)(2 j+\ell+2) e_{j}+\left[(4 j+5) e_{\gamma}-B\right] e_{j+1} \\
& \quad+\left[-\left(j+\frac{5}{2}\right) g_{2}+4 e_{\gamma}^{2}\right](j+2) e_{j+2}-(j+2)(j+3) g_{3} e_{j+3}=0 \\
& (2 j-\ell+2)(2 j+\ell+3) f_{j}+\left[-(4 j+7) e_{\gamma}-B\right] f_{j+1} \\
& \quad+\left[-\left(j+\frac{3}{2}\right) g_{2}-4 e_{\gamma}^{2}\right](j+2) f_{j+2}-(j+2)(j+3) g_{3} f_{j+3}=0
\end{aligned}
$$

It is easy to determine the integers $\ell$ for which $C, D, E, F$ may be a polynomial.

Proposition 3.1. If $\ell \geq 1$ is odd, nonzero Type I Lamé polynomials of the fourth species and Type II ones of the second species can be constructed, with $\operatorname{deg} D=$ 
Table 3. Spectral polynomials for the Lamé polynomial solutions of Types I,II

(Most of the ones with $\ell \geq 5$ disagree with those published by Guerritore (1909).)

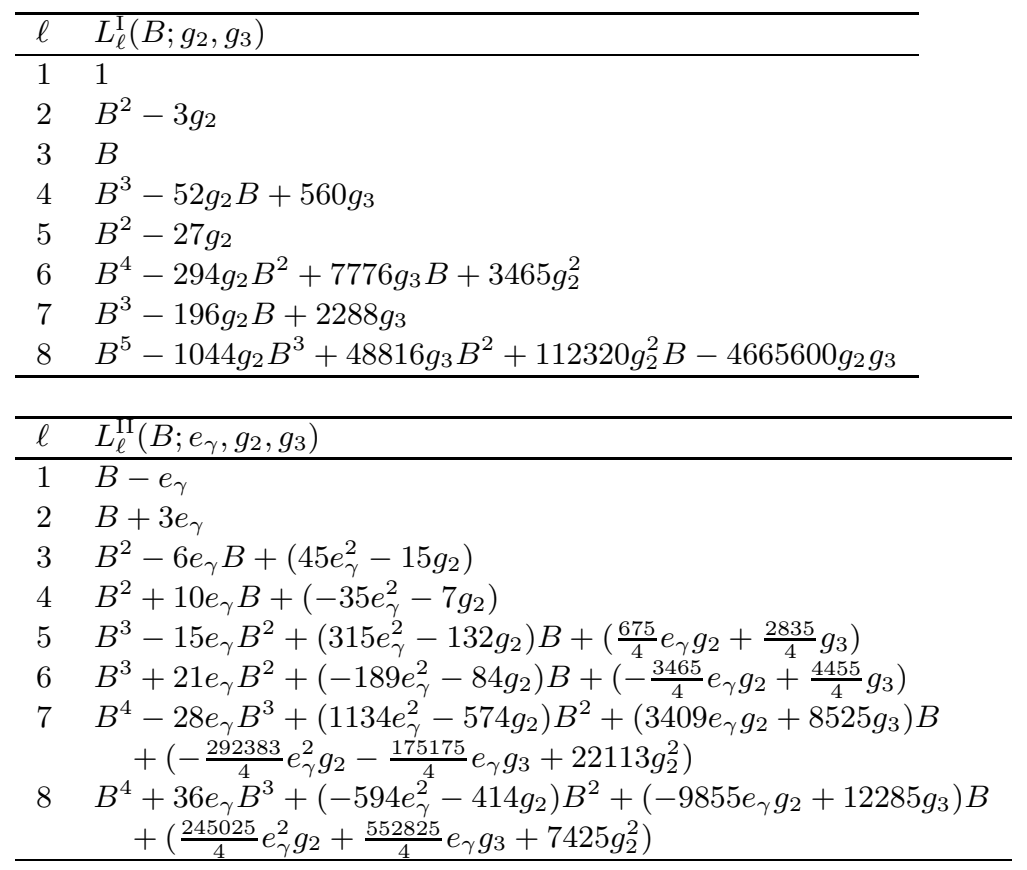

$(\ell-3) / 2$ and $\operatorname{deg} E=(\ell-1) / 2$ respectively. (The former assumes $\ell \geq 3$.) If $\ell \geq 2$ is even, nonzero Type I Lamé polynomial of the first species and Type II ones of the third species can be constructed, with $\operatorname{deg} C=\ell / 2$ and $\operatorname{deg} F=(\ell-2) / 2$ respectively.

The coefficients in each solution can be computed from the recurrence relation by setting the coefficient of the highest power of $x$ to unity, and working downward. Unless $B$ is specially chosen, the coefficients of negative powers of $x$ may be nonzero. But by examination, they will be zero if the coefficient of $x^{-1}$ equals zero.

Definition. The Type-I Lamé spectral polynomial $L_{\ell}^{\mathrm{I}}\left(B ; g_{2}, g_{3}\right)$ is the polynomial monic in $B$ which is proportional to the coefficient $d_{-1}$ if $\ell$ is odd and $c_{-1}$ if $\ell$ is even. (The former assumes $\ell \geq 3$; by convention $L_{1}^{\mathrm{I}} \equiv 1$.) The Type-II Lamé spectral polynomial $L_{\ell}^{\mathrm{II}}\left(B ; e_{\gamma}, g_{2}, g_{3}\right)$ is similarly obtained from the coefficient $e_{-1}$ if $\ell$ is odd and $f_{-1}$ if $\ell$ is even. Each spectral polynomial may be regarded as $\prod_{s}\left[B-B_{s}\left(g_{2}, g_{3}\right)\right]$, resp. $\prod_{s}\left[B-B_{s}\left(e_{\gamma}, g_{2}, g_{3}\right)\right]$, where the roots $\left\{B_{s}\right\}$ are the values of $B$ for which a Lamé equation solution of the indicated type exists, counted with multiplicity.

By examination, $N_{\ell}^{\mathrm{I}} \equiv \operatorname{deg} L_{\ell}^{\mathrm{I}}$ is $(\ell-1) / 2$ if $\ell$ is odd and $\ell / 2+1$ if $\ell$ is even; and $N_{\ell}^{\mathrm{II}} \equiv \operatorname{deg} L_{\ell}^{\mathrm{I}}$ is $(\ell+1) / 2$ if $\ell$ is odd and $\ell / 2$ if $\ell$ is even.

$$
\mathrm{L}_{\ell}\left(B ; g_{2}, g_{3}\right) \equiv L_{\ell}^{\mathrm{I}}\left(B ; g_{2}, g_{3}\right) \prod_{\gamma=1}^{3} L_{\ell}^{\mathrm{II}}\left(B ; e_{\gamma}, g_{2}, g_{3}\right),
$$

the full Lamé spectral polynomial, has degree $N_{\ell}^{\mathrm{I}}+3 N_{\ell}^{\mathrm{II}}=2 \ell+1$ in $B$.

It should be noted that $\prod_{\gamma=1}^{3} L_{\ell}^{\mathrm{II}}\left(B ; e_{\gamma}, g_{2}, g_{3}\right)$, the full Type-II Lamé spectral polynomial, is a function only of $B ; g_{2}, g_{3}$, since any symmetric polynomial 
Table 4. Cohn polynomials for the Lamé polynomial solutions of Types I,II

\begin{tabular}{ll}
\hline$\ell$ & Type I Cohn polynomial \\
\hline 1 & - \\
2 & $J$ \\
3 & - \\
4 & $2^{2} 3^{5} J+5^{2} 7^{2}$ \\
5 & $J$ \\
6 & $2^{4} 3^{2} 5^{2} J^{2}+11 \cdot 37 \cdot 59 J-2^{2} 3^{7}$ \\
7 & $2^{4} 3^{5} 5^{2} J+11^{2} 13^{2}$ \\
8 & $J\left(2^{12} 3^{5} 5^{2} 7^{2} J^{3}+2^{8} 3^{3} 3664447 J^{2}-2^{4} 3^{2} \cdot 397 \cdot 364069 J+113^{5}\right)$ \\
\hline & \\
\hline$\ell$ & Type II Cohn polynomial \\
\hline 1 & - \\
2 & $-\quad$ \\
3 & $2^{2} J+1$ \\
4 & $2^{2} 3^{5} J-5^{3}$ \\
5 & $2^{14} 3^{6} J^{3}+2^{9} 3^{3} 5^{2} 109 J^{2}-2^{2} 5^{4} 17 \cdot 151 J+5^{6} 7^{3}$ \\
6 & $2^{16} 3^{6} 5^{2} J^{3}+2^{11} 3^{3} 17 \cdot 359 J^{2}+2^{4} 57774169 J+33^{3} 109^{3}$ \\
7 & $2^{20} 3^{21} 5^{6} J^{6}+2^{16} 3^{17} 5^{4} 19 \cdot 22307 J^{5}+2^{13} 3^{13} 5^{5} 22158751 J^{4}$ \\
& $\quad+2^{9} 3^{6} 5^{2} 1276543 \cdot 414016613 J^{3}-2^{4} 3^{5} 5^{2} 47202908378639011 J^{2}$ \\
& $\quad+3^{7} 29 \cdot 41 \cdot 101 \cdot 895253 \cdot 8050981 J-2^{5} 5^{6} 11^{3} 37^{3} 113^{3}$ \\
8 & $2^{20} 3^{9} 5^{2} 7^{2} J^{6}+2^{16} 3^{9} 107 \cdot 419 J^{5}+2^{13} 3^{7} 12486499 J^{4}-2^{9} 3^{7} 1171 \cdot 10477 J^{3}$ \\
& $\quad-2^{4} 3^{4} 11 \cdot 47 \cdot 91938173 J^{2}+3^{4} 20593 \cdot 844499 J-2^{5} 7^{3} 13^{3} 29^{3}$ \\
\hline
\end{tabular}

in $e_{1}, e_{2}, e_{3}$ can be written in terms of $g_{2}, g_{3}$. For example, $e_{1} e_{2} e_{3}=g_{3} / 4$. This is why $e_{\gamma}$ is absent on the left-hand side of (3.5). When using the recurrences (3.1)(3.4), one should also note that $4 e_{\gamma}^{3}-g_{2} e_{\gamma}-g_{3}=0$, so $e_{\gamma}^{3}=\frac{1}{4}\left(g_{2} e_{\gamma}+g_{3}\right)$. Any polynomial in $e_{\gamma}, g_{2}, g_{3}$ can be reduced to one which is of degree at most 2 in $e_{\gamma}$, much as any polynomial in $x, y$ can be reduced to one of degree at most 1 in $y$.

The Lamé polynomials of Types I and II are listed in table 2, and the corresponding spectral polynomials in table 3. They replace the table of Guerritore (1909), with its many unfortunate errors. The spectral polynomials with $\ell \leq 7$ were recently computed by a different technique (van der Waall 2002, table A.3). The table of van der Waall displays the full Type II spectral polynomials, rather than the more fundamental $e_{\gamma}$-dependent polynomials $L_{\ell}^{\mathrm{II}}\left(B ; e_{\gamma}, g_{2}, g_{3}\right)$.

The roots of the spectral polynomials are the energies $B$ for which the Lamé polynomials are solutions of the Lamé equation. It is clear that when $\ell \geq 9$, the Type II energies cannot be expressed in terms of radicals, since the degree of the spectral polynomial will be 5 or above. When $\ell=8$ or $\ell \geq 10$, the Type I energies cannot be so expressed. These statements apply also to the coefficients of the Lamé polynomials, which depend on $B$. So when $\ell \geq 10$, the symbolic computation of the Lamé polynomials is impossible, and when $\ell=8$ or 9 , it is possible only in part. But when $g_{2}, g_{3}$ take on special values, what would otherwise be impossible may become possible. For instance, when $g_{3}=0$ (the lemniscatic case, including $m=\frac{1}{2}$ ), the quintic spectral polynomial $L_{8}^{\mathrm{I}}\left(B ; g_{2}, g_{3}\right)$ reduces to $B^{5}-1044 g_{2} B^{3}+112320 g_{2}^{2} B$, the roots of which can obviously be expressed in terms of radicals.

In the context of the Jacobi form, the $2 \ell+1$ values $\left\{E_{s}(m)\right\}_{s=0}^{2 \ell+1}$ for which a Lamé polynomial solution exists can be thought of as the $2 \ell+1$ branches of a 
spectral curve that lies over over the triply-punctured sphere $\mathbb{P}^{1} \backslash\{0,1, \infty\}$, the space of values of the modular parameter $m$. Turbiner (1989) showed that if $\ell \geq 2$, this curve has only four connected components, not $2 \ell+1$. We can now see why. These are the Type I component and the three Type II components, one associated with each point $e_{\gamma}$. Since each of the four is defined by a polynomial in $E$ and $m$, each can be extended to an algebraic curve over $\mathbb{P}^{1}$. At the values $m=0,1, \infty$, the four curves may touch one another. (See, e.g., Li et al. (2000, figure 3), for the behavior of the real portions of the $\ell=1$ and $\ell=2$ curves as $m \rightarrow 0,1$.) These three values of $m$ correspond to two of $e_{1}, e_{2}, e_{3}$ coinciding, and the elliptic curve $y^{2}=4 \prod_{\gamma=1}^{3}\left(x-e_{\gamma}\right)$ becoming rational rather than elliptic. Level crossings of this sort are perhaps less interesting than 'intra-curve' ones.

In the present context, $E$ is replaced by the transformed energy $B$, and $m$ by the pair $g_{2}, g_{3}$ or the Klein invariant $J$, with $J=\infty$ corresponding to $m=0,1, \infty$. It is easy to determine which finite values of $J$ yield coincident values of $B$. One simply computes the discriminants of the Type I and Type II spectral polynomials, $L_{\ell}^{\mathrm{I}}\left(B ; g_{2}, g_{3}\right)$ and $L_{\ell}^{\mathrm{II}}\left(B ; e_{\gamma}, g_{2}, g_{3}\right)$. Each discriminant is zero if and only if there is a double root. By using $J=g_{2}^{3} /\left(g_{2}^{3}-27 g_{3}^{2}\right)$, one can eliminate $g_{2}, g_{3}$ and obtain a polynomial equation for $J$. For each of Types I and II, there are coincident values of $B$ if and only if $J$ is a root of what we call a Cohn polynomial.

In table 4 the Cohn polynomials are listed. Since the coefficients are rather large integers that may have number-theoretic significance, each is given in a fully factored form. An interesting feature of these polynomials is that none has a zero on the real half-line $[1, \infty)$. Since $J \in[1, \infty)$ corresponds to $m \in(0,1)$, the existence of such a zero would imply that for some $m \in(0,1)$, two of the $2 \ell+1$ band edges become degenerate. This is known not to occur (Whittaker \& Watson 1927, §23.41).

The following conjecture is based on a close examination of the spectral and Cohn polynomials, for $\ell$ up to and including $\ell=25$.

Conjecture 3.2. 1. For any integer $\ell \geq 1$, the degeneracies of the algebraic spectrum of the Lamé operator, which comprises the $2 \ell+1$ roots (up to multiplicity) of the spectral polynomial $\mathrm{L}_{\ell}\left(B ; g_{2}, g_{3}\right)$, are fully captured by the Cohn polynomials of Types I and II. As the parameters $g_{2}, g_{3}$ are varied, a pair of roots will coincide, reducing the number of distinct roots from $2 \ell+1$ to $2 \ell$, if and only if the Klein invariant $J$ is a root of one of the two Cohn polynomials; and there are no multiple coincidences.

2. As a polynomial in $J$ with integer coefficients, no Cohn polynomial has a nontrivial factor, except for the Type I polynomials with $\ell \equiv 2(\bmod 3)$, each of which is divisible by $J$. (These factors of $J$ are visible in table 4.)

3. If $N_{\ell}^{\mathrm{I}}$ and $N_{\ell}^{\mathrm{II}}$ denote the degrees of the spectral polynomials of Types I,II, which are given above, then the Cohn polynomials of Types I,II have degrees $\left\lfloor\left(N_{\ell}^{\mathrm{I}^{2}}-N_{\ell}^{\mathrm{I}}+4\right) / 6\right\rfloor$ and $N_{\ell}^{\mathrm{II}}\left(N_{\ell}^{\mathrm{II}}-1\right) / 2$, respectively.

The conjectured degree formulas constitute a conjecture as to the number of points in elliptic moduli space (elliptic curve parameter space), labelled by $J$, at which the $2 \ell+1$ distinct energies in the algebraic spectrum are reduced to $2 \ell$. The remarks regarding extra $J$ factors amount to a conjecture that in the equianharmonic case $m=\frac{1}{2} \pm \frac{\sqrt{3}}{2}$ i (i.e., $J=0$ or $g_{2}=0$ ), there are only $2 \ell$ distinct 
energies if and only if $\ell \equiv 2(\bmod 3)$. For those values of $\ell$, the double energy eigenvalue is evidently located at $B=0$. It should be mentioned that when $J=0$ and $\ell \equiv 0(\bmod 3)$, there is also an eigenvalue at $B=0$, but it is a simple one.

A periodicity of length 3 in $\ell$ is present in the equianharmonic case of a thirdorder equation resembling (2.10), now called the Halphen equation (Halphen 1888, pp 571-4). By the preceding, a similar periodicity appears to be present in the equianharmonic case of the Lamé equation. This was not previously realized.

\section{(b) Twisted Lamé polynomials}

The twisted Lamé polynomials are exponentially modified Lamé polynomials. They will play a major role in Conjecture $\mathrm{L}$ and in the hyperelliptic reductions following from the Hermite-Krichever Ansatz, but they are of independent interest.

Definition. A solution of the Lamé equation (2.9) on the elliptic curve $E_{g_{2}, g_{3}}$ is said to be a twisted Lamé polynomial, of Type I or Type II associated with the point $e_{\gamma}$, if it has one of the two forms

$$
\left\{\begin{array}{c}
C(x)+D(x) y \\
E(x) \sqrt{x-e_{\gamma}}+F(x) y / \sqrt{x-e_{\gamma}}
\end{array}\right\} \times \exp \left[\kappa \int \frac{\mathrm{d} x}{y}\right],
$$

with $\kappa \in \mathbb{C}$ nonzero. Here $C, D, E, F$ are polynomials.

On the level of Fuchsian differential equations, there is little to distinguish between twisted Lamé polynomials and conventional Lamé polynomials, which are simply twisted polynomials with $\kappa=0$. A function $\Psi(x, y)=\widetilde{\Psi}(x, y) \exp \left[\kappa \int \mathrm{d} x / y\right]$ will be a solution of the Lamé equation (2.9) if and only if $\widetilde{\Psi}$ satisfies

$$
\left\{\left(y \frac{\mathrm{d}}{\mathrm{d} x}\right)^{2}+2 \kappa\left(y \frac{\mathrm{d}}{\mathrm{d} x}\right)-\left[\ell(\ell+1) x+B-\kappa^{2}\right]\right\} \widetilde{\Psi}=0 .
$$

This is a Fuchsian equation on $E_{g_{2}, g_{3}}$ that generalizes but strongly resembles (2.9). It has a single regular singular point, $(x, y)=(\infty, \infty)$, and its characteristic exponents there are $\{-\ell, \ell+1\}$. Like $B, \kappa$ is an accessory parameter that does not affect the exponents. The values $(B, \kappa)$ for which a twisted or conventional Lamé polynomial solution of (2.9) exists can be viewed as the points in a two-dimensional accessory parameter space at which (3.6) has single or double-valued solutions.

If $C(x)=\sum_{j} c_{j} x^{j}, D(x)=\sum_{j} d_{j} x^{j}, E(x)=\sum_{j} e_{j} x^{j}$, and $F(x)=\sum_{j} f_{j} x^{j}$, substituting the expression for each type of twisted polynomial solution into (2.9) and equating the coefficients of powers of $x$ yields the coupled pairs of recurrences

$$
\begin{aligned}
& (2 j-\ell)(2 j+\ell+1) c_{j}+\left(\kappa^{2}-B\right) c_{j+1} \\
& \quad-(j+2)\left(j+\frac{3}{2}\right) g_{2} c_{j+2}-(j+2)(j+3) g_{3} c_{j+3} \\
& \quad+2 \kappa(4 j+2) d_{j-1}-2 \kappa\left(j+\frac{3}{2}\right) g_{2} d_{j+1} \\
& \quad-2 \kappa(j+2) g_{3} d_{j+2}=0 \\
& (2 j-\ell+3)(2 j+\ell+4) d_{j}+\left(\kappa^{2}-B\right) d_{j+1} \\
& \quad-(j+2)\left(j+\frac{5}{2}\right) g_{2} d_{j+2}-(j+2)(j+3) g_{3} d_{j+3} \\
& \quad+2 \kappa(j+2) c_{j+2}=0
\end{aligned}
$$




$$
\begin{aligned}
& (2 j-\ell+1)(2 j+\ell+2) e_{j}+\left[(4 j+5) e_{\gamma}+\kappa^{2}-B\right] e_{j+1} \\
& \quad+\left[-\left(j+\frac{5}{2}\right) g_{2}+4 e_{\gamma}^{2}\right](j+2) e_{j+2}-(j+2)(j+3) g_{3} e_{j+3} \\
& \quad+2 \kappa(4 j+4) f_{j}+2 \kappa(4 j+6) e_{\gamma} f_{j+1} \\
& \quad+2 \kappa(4 j+8)\left(e_{\gamma}^{2}-\frac{1}{4} g_{2}\right) f_{j+2}=0 \\
& (2 j-\ell+2)(2 j+\ell+3) f_{j}+\left[-(4 j+7) e_{\gamma}+\kappa^{2}-B\right] f_{j+1} \\
& \quad+\left[-\left(j+\frac{3}{2}\right) g_{2}-4 e_{\gamma}^{2}\right](j+2) f_{j+2}-(j+2)(j+3) g_{3} f_{j+3} \\
& \quad+2 \kappa\left(j+\frac{3}{2}\right) e_{j+1}-2 \kappa(j+2) e_{\gamma} e_{j+2}=0
\end{aligned}
$$

It is easy to determine the maximum value of the exponent $j$ in each of $C, D, E, F$.

Proposition 3.3. Nonzero twisted Lamé polynomials of Type $I$ (if $\ell \geq 3$ ) and of Type II (if $\ell \geq 2)$ can in principle be constructed from these recurrence relations. If $\ell$ is odd, resp. even, then $\operatorname{deg} C, D ; E, F$ are $(\ell-1) / 2,(\ell-3) / 2 ;(\ell-1) / 2,(\ell-3) / 2$, resp. $\ell / 2,(\ell-4) / 2 ; \ell / 2-1, \ell / 2-1$. The coefficients can be computed by setting the coefficient of the highest power of $x$ to unity in $D$ and $E$, resp. $C$ and $F$, and working downward.

Unless $B, \kappa$ are specially chosen, the coefficients of negative powers of $x$ may be nonzero. But by examination, they will be zero if the coefficients of $x^{-1}$ in $C$ and $D$ (for Type I), or $E$ and $F$ (for Type II), equal zero. $c_{-1}=0, d_{-1}=0$, and $e_{-1}=0, f_{-1}=0$, are coupled polynomial equations in $B, \kappa$, and their solutions may be computed by polynomial elimination, e.g., by computing resultants. A minor problem is the proper handling of the case $\kappa=0$, in which (3.7)-(3.10) reduce to (3.1)-(3.4). If $\ell$ is odd, resp. even, then $c_{-1}$ and $f_{-1}$, resp. $d_{-1}$ and $e_{-1}$, turn out to be divisible by $\kappa$. By dividing the appropriate equations by $\kappa$ before solving each pair of coupled equations, the spurious $\kappa=0$ solutions can be eliminated.

Definition. The Type-I twisted Lamé spectral polynomial $L t_{\ell}^{\mathrm{I}}\left(B ; g_{2}, g_{3}\right)$ is the polynomial monic in $B$ which is proportional to the resultant of $c_{-1}, d_{-1}$ with respect to $\kappa$, with $\kappa$ factors removed as indicated. (This assumes $\ell \geq 3$; by convention $L t_{1}^{\mathrm{I}}=L t_{2}^{\mathrm{I}} \equiv 1$.) The Type-II twisted Lamé spectral polynomial $L t_{\ell}^{\mathrm{II}}\left(B ; e_{\gamma}, g_{2}, g_{3}\right)$ is similarly obtained from $e_{-1}, f_{-1}$. (This assumes $\ell \geq 2$; by convention $L t_{1}^{\mathrm{II}} \equiv 1$.) Each twisted spectral polynomial may be regarded as $\prod_{s}\left[B-B_{s}\left(g_{2}, g_{3}\right)\right]$, resp. $\prod_{s}\left[B-B_{s}\left(e_{\gamma}, g_{2}, g_{3}\right)\right]$, where the roots $\left\{B_{s}\right\}$ are the values of $B$ for which a Lamé equation solution of the specified type exists, counted with multiplicity.

The twisted Lamé spectral polynomials for $\ell \leq 8$ are listed in table 5 . The polynomials $L t_{7}^{\mathrm{II}}$ and $L t_{8}^{\mathrm{II}}$ are omitted due to lack of space (their respective degrees are 12 and 16). The following conjecture is based on an extensive computation of $L t_{\ell}^{\mathrm{I}}$ and $L t_{\ell}^{\mathrm{II}}$, at these and higher values of $\ell$.

Conjecture 3.4. 1. For any integer $\ell \geq 3$, resp. $\ell \geq 2$, there is a nontrivial twisted spectral polynomial of Type I, resp. Type II.

2. For any integer $\ell \geq 1, N t_{\ell}^{\mathrm{I}} \equiv \operatorname{deg} L t_{\ell}^{\mathrm{I}}$ is $\left(\ell^{2}-1\right) / 4$ if $\ell$ is odd and $\ell^{2} / 4-1$ if $\ell$ is even; and $N t_{\ell}^{\mathrm{II}} \equiv \operatorname{deg} L t_{\ell}^{\mathrm{II}}$ is $\left(\ell^{2}-1\right) / 4$ if $\ell$ is odd and $\ell^{2} / 4$ if $\ell$ is even.

If the conjectured degree formulas are true, then the full twisted Lamé spectral polynomial $\mathrm{Lt}_{\ell}\left(B ; g_{2}, g_{3}\right) \equiv L t_{\ell}^{\mathrm{I}}\left(B ; g_{2}, g_{3}\right) \prod_{\gamma=1}^{3} L t_{\ell}^{\mathrm{II}}\left(B ; e_{\gamma}, g_{2}, g_{3}\right)$ will be of degree 
Table 5. Spectral polynomials for the twisted Lamé polynomial solutions of Types I,II

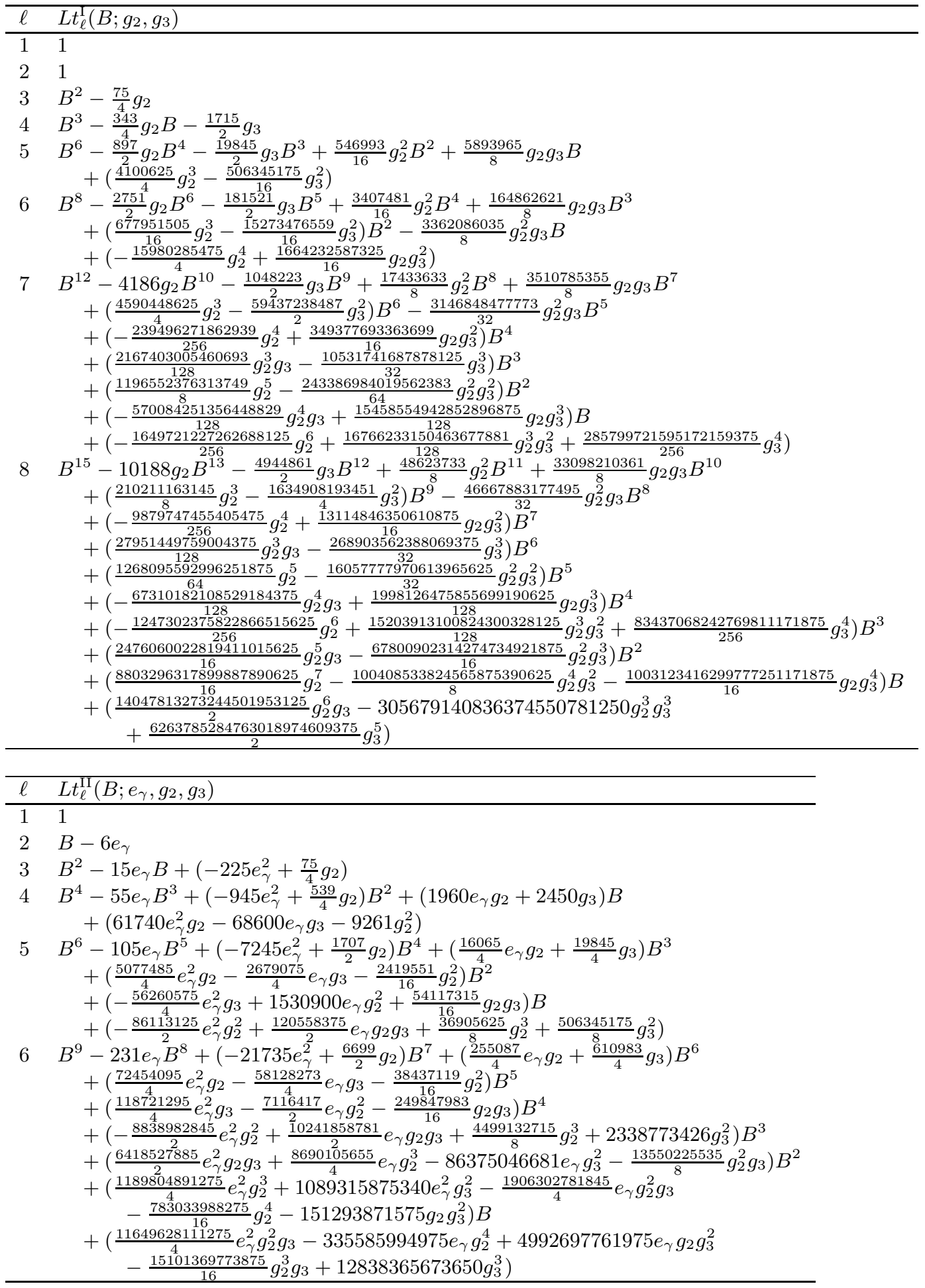


$N t_{\ell}^{\mathrm{I}}+3 N t_{\ell}^{\mathrm{II}}=\ell^{2}-1$ in the energy parameter $B$. Like the standard degree- $(2 \ell+1)$ spectral polynomial $\mathrm{L}_{\ell}, \mathrm{Lt}_{\ell}$ is a function of $B ; g_{2}, g_{3}$ only, because any symmetric polynomial in $e_{1}, e_{2}, e_{3}$ can be written in terms of the invariants $g_{2}, g_{3}$.

\section{(c) Theta-twisted Lamé polynomials}

Lamé equation solutions of a third sort can be constructed for certain values of the energy parameter $B$. These are linear combinations, over polynomials in the coordinate $x$, of (i) the multi-valued meromorphic function $\Phi\left(x, y ; x_{0}, y_{0}\right)$ parametrized by the point $\left(x_{0}, y_{0}\right) \in E_{g_{2}, g_{3}} \backslash\{(\infty, \infty)\}$, and (ii) its derivative

$$
\Phi^{(1)}\left(x, y ; x_{0}, y_{0}\right) \equiv\left(y \frac{\mathrm{d}}{\mathrm{d} x}\right) \Phi\left(x, y ; x_{0}, y_{0}\right)=\frac{1}{2}\left(\frac{y+y_{0}}{x-x_{0}}\right) \Phi\left(x, y ; x_{0}, y_{0}\right) .
$$

One way of seeing that $\Phi, \Phi^{(1)}$ are a natural basis is to note that when $\left(x_{0}, y_{0}\right)=$ $\left(e_{\gamma}, 0\right)$, they reduce to $\sqrt{x-e_{\gamma}}, \frac{1}{2} y / \sqrt{x-e_{\gamma}}$. So the class of functions constructed from them will include the Lamé polynomials of Type II.

Definition. A solution of the Lamé equation (2.9) on the elliptic curve $E_{g_{2}, g_{3}}$ is said to be a theta-twisted Lamé polynomial if it is of the form $\mathcal{A}(x) \Phi\left(x, y ; x_{0}, y_{0}\right)+$ $2 \mathcal{B}(x) \Phi^{(1)}\left(x, y ; x_{0}, y_{0}\right)$, with $\left(x_{0}, y_{0}\right) \neq\left(e_{\gamma}, 0\right)$ for $\gamma=1,2,3$. Here $\mathcal{A}, \mathcal{B}$ are polynomials, and the innocuous ' 2 ' factor compensates for the ' $\frac{1}{2}$ ' factor of (3.11).

If $\mathcal{A}(x)=\sum_{j} a_{j} x^{j}$ and $\mathcal{B}(x)=\sum_{j} b_{j} x^{j}$, substituting this expression into (2.9) and equating the coefficients of powers of $x$ yields the coupled pair of recurrences

$$
\begin{aligned}
& (2 j-\ell+1)(2 j+\ell+2) a_{j}+\left[(4 j+5) x_{0}-B\right] a_{j+1} \\
& \quad+\left[-\left(j+\frac{5}{2}\right) g_{2}+4 x_{0}^{2}\right](j+2) a_{j+2}-(j+2)(j+3) g_{3} a_{j+3} \\
& \quad-2 y_{0}(4 j+6) b_{j+1}-4 x_{0} y_{0}(j+2) b_{j+2}=0 \\
& (2 j-\ell+2)(2 j+\ell+3) b_{j}+\left[-(4 j+7) x_{0}-B\right] b_{j+1} \\
& \quad+\left[-\left(j+\frac{3}{2}\right) g_{2}-4 x_{0}^{2}\right](j+2) b_{j+2}-(j+2)(j+3) g_{3} b_{j+3} \\
& \quad+y_{0}(j+2) a_{j+2}=0
\end{aligned}
$$

Proposition 3.5. If $\ell \geq 4$, nonzero theta-twisted polynomials can in principle be computed from these recurrences. If $\ell$ is odd, resp. even, then $\operatorname{deg} \mathcal{A}, \mathcal{B}$ are $(\ell-1) / 2$, $(\ell-5) / 2$, resp. $\ell / 2-2, \ell / 2-1$. The coefficients can be computed by setting the coefficient of the highest power of $x$ in $\mathcal{A}$, resp. $\mathcal{B}$, to unity, and working downward.

Unless $B$ and the point $\left(x_{0}, y_{0}\right)$ are specially chosen, the coefficients of negative powers of $x$ may be nonzero. But by examination, they will be zero if the coefficients of $x^{-1}$ in $\mathcal{A}$ and $\mathcal{B}$ are both zero. $a_{-1}=0, b_{-1}=0$ are equations in $B ; x_{0}, y_{0}$. Together with the identity $y_{0}^{2}=4 x_{0}^{3}-g_{2} x_{0}-g_{3}$, they make up a set of three polynomial equations for these three unknowns. This system may be solved by polynomial elimination. For example, to obtain a single polynomial equation for $B$ (involving $g_{2}, g_{3}$ of course), one may eliminate $y_{0}$ from $a_{-1}=0, b_{-1}=0$ by computing their resultants against the third equation; and then eliminate $x_{0}$. Alternatively, a Gröbner basis calculation may be performed (Brezhnev 2000).

Irrespective of which procedure is followed, there is a minor problem: the handling of the improper case $\left(x_{0}, y_{0}\right)=\left(e_{\gamma}, 0\right)$, in which (3.12)-(3.13) reduce to (3.3)(3.4). If $\ell$ is odd, resp. even, then the left-hand side of the equation $b_{-1}=0$, resp. 
Table 6. Spectral polynomials for the theta-twisted Lamé polynomial solutions

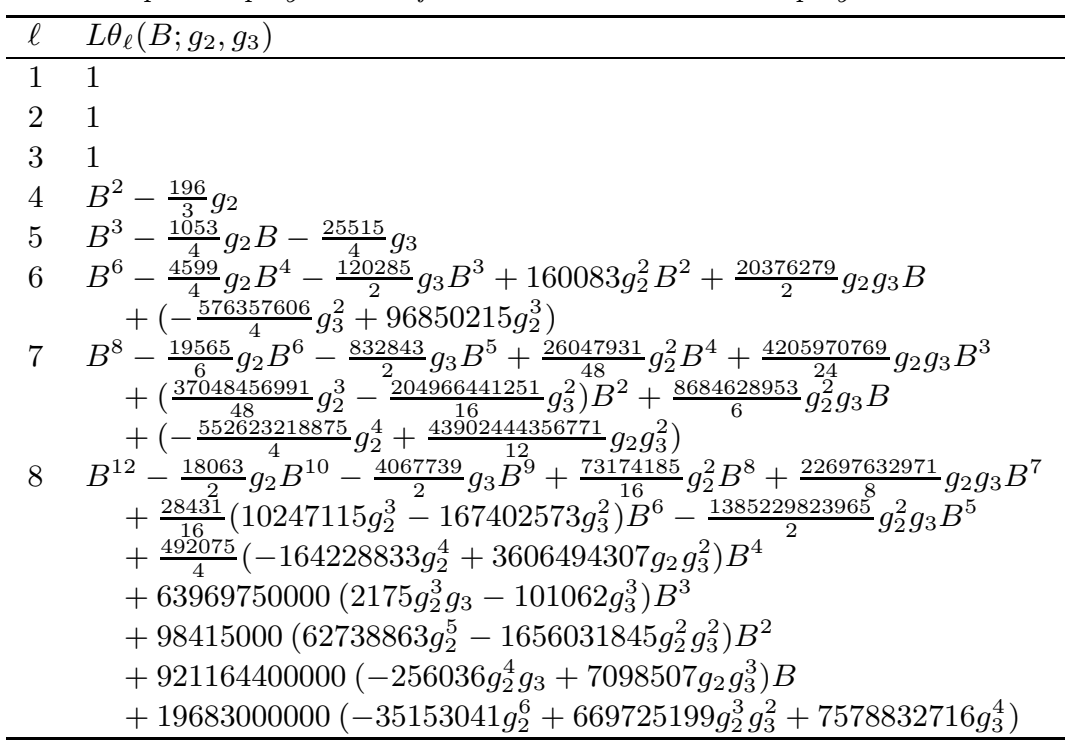

$a_{-1}=0$, turns out to be divisible by $y_{0}$. By dividing the appropriate equation by $y_{0}$ before eliminating $x_{0}, y_{0}$, the spurious solutions with $y_{0}=0$ can be eliminated.

Definition. The theta-twisted Lamé spectral polynomial $L \theta_{\ell}\left(B ; g_{2}, g_{3}\right)$ is the polynomial monic in $B$ which is obtained by eliminating $x_{0}, y_{0}$ from the equations $a_{-1}=0, b_{-1}=0$, with $y_{0}$ factors removed as indicated. (This assumes $\ell \geq 4$; by convention $L \theta_{1}=L \theta_{2}=L \theta_{3} \equiv 1$.) Each theta-twisted spectral polynomial may be regarded as $\prod_{s}\left[B-B_{s}\left(g_{2}, g_{3}\right)\right]$, where the roots $\left\{B_{s}\right\}$ are the values of $B$ for which a theta-twisted Lamé polynomial exists, counted with multiplicity.

The theta-twisted Lamé spectral polynomials for $\ell \leq 8$ are listed in table 6 . The following conjecture is based on these polynomials, and on higher ones.

Conjecture 3.6. 1. For any integer $\ell \geq 4$ there is a nontrivial theta-twisted spectral polynomial $L \theta_{\ell}$.

2. For any integer $\ell \geq 2, N \theta_{\ell} \equiv \operatorname{deg} L \theta_{\ell}$ is $(\ell+1)(\ell-3) / 4$ if $\ell$ is odd and $\ell(\ell-2) / 4$ if $\ell$ is even.

\section{The Hermite-Krichever Ansatz}

The Hermite-Krichever Ansatz may be applied to any Schrödinger-like differential equation, not necessarily of second order, with coefficient functions that are elliptic. According to the Ansatz, any such equation should ideally have one or more independent solutions which can be expressed as finite series in the derivatives of an elliptic Baker-Akhiezer function, multiplied by an exponential function. (Cf. (1.5).)

Applied to (2.9), the elliptic-curve algebraic form of the Lamé equation, the Ansatz asserts that except at isolated values of the energy $B \in \mathbb{C}$, it should be possible to construct a solution on the curve $E_{g_{2}, g_{3}}$ as a finite series in the multivalued functions $\Phi^{(j)} \equiv(y \mathrm{~d} / \mathrm{d} x)^{j} \Phi, j \geq 0$, multiplied by a factor $\exp \left[\kappa \int \mathrm{d} x / y\right]$. 
Here $\Phi\left(\cdot, \cdot ; x_{0}, y_{0}\right)$ is the fundamental multi-valued meromorphic function on $E_{g_{2}, g_{3}}$ introduced in $\S 2$. Actually, a different but equivalent sort of series is easier to manipulate symbolically. By examination $\Phi^{(2)}=\left(2 x+x_{0}\right) \Phi$, from which it follows by induction on $j$ that any finite series in $\Phi^{(j)}, j \geq 0$, is a combination (over polynomials in $x$ ) of the basis functions $\Phi, \Phi^{(1)}$. This motivates the following definition.

Definition. A solution of the Lamé equation (2.9) on the elliptic curve $E_{g_{2}, g_{3}}$ is said to be an Hermite-Krichever solution if it is of the form

$$
\left[\mathcal{A}(x) \Phi\left(x, y ; x_{0}, y_{0}\right)+2 \mathcal{B}(x) \Phi^{(1)}\left(x, y ; x_{0}, y_{0}\right)\right] \exp \left[\kappa \int \frac{\mathrm{d} x}{y}\right]
$$

for some $\left(x_{0}, y_{0}\right) \in E_{g_{2}, g_{3}} \backslash\{(\infty, \infty)\}$ and $\kappa \in \mathbb{C}$. Here $\mathcal{A}, \mathcal{B}$ are polynomials.

As defined, Hermite-Krichever solutions subsume the solutions explored in $\S 3$. If $\kappa=0$, they reduce to theta-twisted Lamé polynomials. If $\left(x_{0}, y_{0}\right)=\left(e_{\gamma}, 0\right)$ for $\gamma=1,2,3$, in which case $\Phi, \Phi^{(1)}$ degenerate to $\sqrt{x-e_{\gamma}}, \frac{1}{2} y / \sqrt{x-e_{\gamma}}$, they reduce to twisted Lamé polynomials of Type II. If both specializations occur, they reduce to ordinary Lamé polynomials of Type II. The Lamé polynomials of Type I can be viewed as arising from a passage to the limit $\left(x_{0}, y_{0}\right) \rightarrow(\infty, \infty)$.

If $\mathcal{A}(x)=\sum_{j} a_{j} x^{j}$ and $\mathcal{B}(x)=\sum_{j} b_{j} x^{j}$, substituting (4.1) into (2.9) and equating the coefficients of powers of $x$ yields the coupled pair of recurrences

$$
\begin{aligned}
(2 j- & \ell+1)(2 j+\ell+2) a_{j}+\left[(4 j+5) x_{0}+\kappa^{2}-B\right] a_{j+1} \\
& +\left[-\left(j+\frac{5}{2}\right) g_{2}+4 x_{0}^{2}-2 \kappa y_{0}\right](j+2) a_{j+2}-(j+2)(j+3) g_{3} a_{j+3} \\
& +8 \kappa(j+1) b_{j}+4\left(\kappa x_{0}-y_{0}\right)(2 j+3) b_{j+1} \\
& +2\left[\kappa\left(4 x_{0}^{2}-g_{2}\right)-2 x_{0} y_{0}\right](j+2) b_{j+2}=0 \\
& \quad 2 j-\ell+2)(2 j+\ell+3) b_{j}+\left[-(4 j+7) x_{0}+\kappa^{2}-B\right] b_{j+1} \\
& +\left[-\left(j+\frac{3}{2}\right) g_{2}-4 x_{0}^{2}+2 \kappa y_{0}\right](j+2) b_{j+2}-(j+2)(j+3) g_{3} b_{j+3} \\
& +\kappa(2 j+3) a_{j+1}-\left(2 \kappa x_{0}-y_{0}\right)(j+2) a_{j+2}=0
\end{aligned}
$$

If $\kappa=0,(4.2)-(4.3)$ reduce to $(3.12)-(3.13)$, and if $\left(x_{0}, y_{0}\right)=\left(e_{\gamma}, 0\right)$, they reduce to $(3.9)-(3.10)$. If both specializations occur, they reduce to $(3.3)-(3.4)$.

Proposition 4.1. For all $\ell \geq 2$, Hermite-Krichever solutions can in principle be computed from these recurrences. If $\ell$ is odd, resp. even, then $\operatorname{deg} \mathcal{A}, \mathcal{B}$ are $(\ell-1) / 2$, $(\ell-3) / 2$, resp. $\ell / 2-1, \ell / 2-1$. The coefficients can be computed by setting the coefficient of the highest power of $x$ in $\mathcal{A}$, resp. $\mathcal{B}$, to unity, and working downward.

Unless $B, \kappa$ and the point $\left(x_{0}, y_{0}\right)$ are specially chosen, the coefficients of negative powers of $x$ may be nonzero. But by examination, they will be zero if the coefficients of $x^{-1}$ in $\mathcal{A}$ and $\mathcal{B}$ are both zero. $a_{-1}=0, b_{-1}=0$ are equations in $B ; \kappa ; x_{0}, y_{0}$. They are 'compatibility conditions' similar to those that appear in other applications of the Hermite-Krichever Ansatz. Together with the identity $y_{0}^{2}=4 x_{0}^{3}-g_{2} x_{0}-g_{3}$, they make up a set of three equations for these four unknowns.

Informally, one can eliminate any two of $B ; \kappa ; x_{0}, y_{0}$, and derive an algebraic relation between the remaining two variables (involving $g_{2}, g_{3}$ of course). A rigorous investigation must be more careful. For example, if the ideal generated by 
the three equations contains a polynomial involving only $B$ (and $g_{2}, g_{3}$ ), then the Hermite-Krichever Ansatz will not apply for arbitrary values of $B$ (Brezhnev 2000). In practice, this problem seems not to arise. For $\ell \leq 5$ at least, previous work has shown that an algebraic curve in $(B, \kappa)$ can be derived. This is the curve with the most physical significance, since $B$ is a transformed energy and $\kappa$ is related to the crystal momentum. It appears always to be the case that $x_{0}$ is a rational function of $B$, and $y_{0}$ a rational function of $B$, times $\kappa$. These results can be reformulated in terms of the following algebraic curve.

Definition. The $\ell$ 'th Lamé spectral curve $\Gamma_{\ell} \equiv \Gamma_{\ell}\left(g_{2}, g_{3}\right)$ is the hyperelliptic curve over $\mathbb{P}^{1} \ni B$ comprising all $(B, \nu)$ satisfying $\nu^{2}=\mathrm{L}_{\ell}\left(B ; g_{2}, g_{3}\right)$, where the righthand side is the full Lamé spectral polynomial, of degree $2 \ell+1$. ( $\Gamma_{\ell}$ was informally introduced as $\widetilde{\Gamma}_{\ell}$ in section 1 , where the original energy parameter $E$ was used.) $\Gamma_{\ell}$ will have genus $\ell$ unless two of the roots of $\mathrm{L}_{\ell}\left(\cdot ; g_{2}, g_{3}\right)$ coincide, i.e., unless the Klein invariant $J$ is a root of one of the two Cohn polynomials shown in table 4 , in which case the genus is reduced by 1 .

One expects that Lamé equation solutions can be parametrized by the point $(B, \nu) \in \Gamma_{\ell}$, since that parametrization arises in the theory of Hill's equation on $\mathbb{R}$ (McKean \& van Moerbeke 1979). For $2 \leq \ell \leq 5$ it was discovered that the algebraic curve in $(B, \kappa)$ is isomorphic to $\Gamma_{\ell}$ via a birational transformation of a simple kind: the ratio $\kappa / \nu$ is a rational function of $B$. Due to this, if the point $\left(x_{0}, y_{0}\right)$ is viewed as a function of $(B, \nu)$ via a covering map $\pi_{\ell}: \Gamma_{\ell} \rightarrow E_{g_{2}, g_{3}}$, then $x_{0}$ will be a rational function of $B$, and $y_{0}$ will be a rational function of $B$, times $\nu$.

Even with the aid of computer algebra systems, these functions are difficult to compute. Until recently, the largest value of $\ell$ successfully dealt with was $\ell=5$. With the aid of the MACsYMA system, we have computed them for higher values of $\ell$, and have noticed a regular pattern that previous authors seem to have missed.

Conjecture L. For all integer $\ell \geq 1$, the covering map $\pi_{\ell}: \Gamma_{\ell}\left(g_{2}, g_{3}\right) \rightarrow E_{g_{2}, g_{3}}$ projects $(B, \nu)$ to $\left(x_{0}, y_{0}\right)$ according to

$$
\begin{aligned}
x_{0}\left(B ; g_{2}, g_{3}\right) & =e_{\gamma}+\frac{4}{[\ell(\ell+1)]^{2}} \frac{L_{\ell}^{\mathrm{II}}\left(B ; e_{\gamma}, g_{2}, g_{3}\right)\left[L t_{\ell}^{\mathrm{II}}\left(B ; e_{\gamma}, g_{2}, g_{3}\right)\right]^{2}}{L_{\ell}^{\mathrm{I}}\left(B ; g_{2}, g_{3}\right)\left[L t_{\ell}^{\mathrm{I}}\left(B ; g_{2}, g_{3}\right)\right]^{2}} \\
y_{0}\left(B, \nu ; g_{2}, g_{3}\right) & =\frac{16}{[\ell(\ell+1)]^{3}}\left\{\frac{\prod_{\gamma=1}^{3} L t_{\ell}^{\mathrm{II}}\left(B ; e_{\gamma}, g_{2}, g_{3}\right)}{\left[L_{\ell}^{\mathrm{I}}\left(B ; g_{2}, g_{3}\right)\right]^{2}\left[L t_{\ell}^{\mathrm{I}}\left(B ; g_{2}, g_{3}\right)\right]^{3}}\right\} \nu,
\end{aligned}
$$

with $\gamma$ in (4.4) being any of 1,2,3. Moreover, the function $\kappa: \Gamma_{\ell} \rightarrow \mathbb{P}^{1}$ is given by

$$
\kappa\left(B, \nu ; g_{2}, g_{3}\right)=-\frac{(\ell-1)(\ell+2)}{\ell(\ell+1)}\left[\frac{L \theta_{\ell}\left(B ; g_{2}, g_{3}\right)}{L_{\ell}^{\mathrm{I}}\left(B ; g_{2}, g_{3}\right) L t_{\ell}^{\mathrm{I}}\left(B ; g_{2}, g_{3}\right)}\right] \nu .
$$

These remarkably simply formulas have been verified to be correct for all $\ell \leq 8$, though at present they lack a rigorous justification. They permit Hermite-Krichever solutions of the form (4.1) to be constructed for quite large values of $\ell$, since the spectral polynomials $L, L t, L \theta$ (ordinary, twisted and theta-twisted) are relatively easy to work out, as $\S 3$ made clear. Tables 3,5 and 6 may be consulted.

It should be stressed that in the formula (4.4) for $x_{0}$, the same right-hand side results, irrespective of which of the three values of $\gamma$ is chosen. All terms explicitly 
involving $e_{\gamma}$ will cancel. Of course, all powers of $e_{\gamma}$ higher than the second must first be rewritten in terms of $g_{2}, g_{3}$ by using the identity $e_{\gamma}^{3}=\frac{1}{4}\left(g_{2} e_{\gamma}+g_{3}\right)$. In the same way, it is understood that the numerator of the right-hand side of (4.5), the terms of which are symmetric in $e_{1}, e_{2}, e_{3}$, should be rewritten in terms of $g_{2}, g_{3}$. (This can always be done: for example, $e_{1}^{2} e_{2}^{2}+e_{2}^{2} e_{3}^{2}+e_{3}^{2} e_{1}^{2}$ equals $g_{2}^{2} / 16$.) Incidentally, it is possible that previous authors missed the simple expression (4.4) because they attempted to factor the rational expression for $x_{0}(B)$, rather than that for $x_{0}(B)-e_{\gamma}$. The numerator of the former may be complicated, with no nontrivial factors; but the numerator of the latter, when factored, is readily recognized as being proportional to $L_{\ell}^{\mathrm{II}}(B)\left[L t_{\ell}^{\mathrm{II}}(B)\right]^{2}$. This observation led directly to Conjecture L.

The application of the formulas in Conjecture $\mathrm{L}$ to the cases $\ell=1,2,3$ may be illuminating.

○ If $\ell=1$, then $\left(x_{0}, y_{0}\right)=(B, 2 \nu)$ and $\kappa=0$. The map $\pi_{1}: \Gamma_{1} \rightarrow E_{g_{2}, g_{3}}$ is a mere change of normalization, since $\Gamma_{1}$ is isomorphic to $E_{g_{2}, g_{3}}$; cf. (2.14).

$\circ$ If $\ell=2$, then

$$
\begin{aligned}
& x_{0}=e_{\gamma}+\frac{1}{9} \frac{\left(B+3 e_{\gamma}\right)\left(B-6 e_{\gamma}\right)^{2}}{B^{2}-3 g_{2}}=\frac{B^{3}+27 g_{3}}{9\left(B^{2}-3 g_{2}\right)}, \\
& y_{0}=\frac{2}{27} \frac{\prod_{\gamma=1}^{3}\left(B-6 e_{\gamma}\right)}{\left(B^{2}-3 g_{2}\right)^{2}} \nu=\frac{2\left(B^{3}-9 g_{2} B-54 g_{3}\right)}{27\left(B^{2}-3 g_{2}\right)^{2}} \nu,
\end{aligned}
$$

and $\kappa=-\left\{2 /\left[3\left(B^{2}-3 g_{2}\right)\right]\right\} \nu$.

○ If $\ell=3$, then

$$
\begin{aligned}
x_{0} & =e_{\gamma}+\frac{1}{36} \frac{\left(B^{2}-6 e_{\gamma} B+45 e_{\gamma}^{2}-15 g_{2}\right)\left(B^{2}-15 e_{\gamma} B-225 e_{\gamma}^{2}+\frac{75}{4} g_{2}\right)^{2}}{B\left(B^{2}-\frac{75}{4} g_{2}\right)^{2}} \\
& =\frac{\left(\begin{array}{c}
16 B^{6}+360 g_{2} B^{4}+27000 g_{3} B^{3}-3375 g_{2}^{2} B^{2} \\
-303750 g_{2} g_{3} B-84375 g_{2}^{3}+2278125 g_{3}^{2}
\end{array}\right)}{36 B\left(4 B^{2}-75 g_{2}\right)^{2}} \\
y_{0} & =\frac{1}{108} \frac{\prod_{\gamma=1}^{3}\left(B^{2}-15 e_{\gamma} B-225 e_{\gamma}^{2}+\frac{75}{4} g_{2}\right)}{B^{2}\left(B^{2}-\frac{75}{4} g_{2}\right)^{3}} \nu \\
& =\frac{\left(\begin{array}{c}
16 B^{6}-1800 g_{2} B^{4}-54000 g_{3} B^{3} \\
-16875 g_{2}^{2} B^{2}+421875 g_{2}^{3}-11390625 g_{3}^{2}
\end{array}\right)}{27 B^{2}\left(4 B^{2}-75 g_{2}\right)^{3}} \nu
\end{aligned}
$$

and $\kappa=-\left\{10 /\left[B\left(12 B^{2}-225 g_{2}\right)\right]\right\} \nu$.

The formulas for $\ell=2,3$ were essentially known to Hermite. Setting $\ell=4,5$ in Conjecture L yields the less familiar and much more complicated formulas that Enol'skii \& Kostov (1994) and Eilbeck \& Enol'skii (1994) derived by eliminating variables from compatibility conditions. The computation of the covering map $\pi_{5}$ by Eilbeck \& Enol'skii required seven hours of computer time, but the present approach requires only a fraction of a second. Assuming the validity of Conjecture L, one can readily compute the map $\pi_{\ell}$ and the auxiliary function $\kappa$ for far larger $\ell$. 


\section{Hyperelliptic reductions}

The cover $\pi_{\ell}: \Gamma_{\ell}\left(g_{2}, g_{3}\right) \rightarrow E_{g_{2}, g_{3}}$ introduced as part of the Hermite-Krichever Ansatz, i.e., the map $(B, \nu) \mapsto\left(x_{0}, y_{0}\right)$, is of independent interest, since explicit examples of coverings of elliptic curves by higher-genus algebraic curves are few, and the problem of determining which curves can cover $E_{g_{2}, g_{3}}$, for either specified or arbitrary values of the invariants $g_{2}, g_{3}$, remains unsolved. $\Gamma_{\ell}\left(g_{2}, g_{3}\right)$ generically has genus $g=\ell$, as noted, and the formula for $x_{0}=x_{0}\left(B ; g_{2}, g_{3}\right)$ given in Conjecture $\mathrm{L}$ implies that the cover will be of degree $N=\ell(\ell+1) / 2$. This is because $N$ equals $\max \left(N_{\ell}^{\mathrm{I}}+2 N t_{\ell}^{\mathrm{I}}, N_{\ell}^{\mathrm{II}}+2 N t_{\ell}^{\mathrm{II}}\right)$, the maximum of the degrees in $B$ of the numerator and denominator of $x_{0}$. The degrees $N_{\ell}^{\mathrm{I}}, N_{\ell}^{\mathrm{II}}$ are known, and conjectured formulas for the twisted degrees $N t_{\ell}^{\mathrm{I}}, N t_{\ell}^{\mathrm{II}}$ were given in conjecture 3.4.

Since $\Gamma_{\ell}\left(g_{2}, g_{3}\right)$ is hyperelliptic (defined by the irrationality $\nu^{2}=\mathrm{L}_{\ell}\left(B ; g_{2}, g_{3}\right)$ ) and $E_{g_{2}, g_{3}}$ is elliptic (defined by the irrationality $y_{0}^{2}=4 x_{0}^{3}-g_{2} x_{0}-g_{3}$ ), the map $\pi_{\ell}$ enables certain hyperelliptic integrals to be reduced to elliptic ones. Just as $E_{g_{2}, g_{3}}$ is equipped with the canonical holomorphic 1 -form $\mathrm{d} x_{0} / y_{0}$, so can $\Gamma_{\ell}\left(g_{2}, g_{3}\right)$ be equipped with the holomorphic 1-form $\mathrm{d} B / \nu$. Any integral of a function in the function field of a hyperelliptic curve (here, any rational function $R(B, \nu)$ ) against its canonical 1-form is called a hyperelliptic integral. Hyperelliptic integrals are classified as follows (Belokolos et al. 1986). The linear space of meromorphic 1-forms of the form $R(B, \nu) \mathrm{d} B / \nu$, i.e., of Abelian differentials, is generated by 1-forms of the first, second and third kinds. These are (i) holomorphic 1-forms, with no poles; (ii) 1-forms with one multiple pole; and (iii) 1-forms with a pair of simple poles, the residues of which are opposite in sign. The indefinite integrals of (i)-(iii) are called hyperelliptic integrals of the first, second and third kinds. They generalize the three kinds of elliptic integral (Abramowitz \& Stegun 1965, chapter 17).

Hyperelliptic integrals of the first kind are the easiest to study, since the linear space of holomorphic 1-forms is finite-dimensional and is spanned by $B^{r-1} \mathrm{~d} B / \nu$, $r=1, \ldots, g$, where $g$ is the genus. So there are only $g$ independent integrals of the first kind. A consequence of the map $\pi_{\ell}: \Gamma_{\ell}\left(g_{2}, g_{3}\right) \rightarrow E_{g_{2}, g_{3}}$ is that on any hyperelliptic curve of the form $\Gamma_{\ell}\left(g_{2}, g_{3}\right)$ there are really only $g-1$ independent integrals of the first kind, modulo elliptic integrals (considered trivial by comparison). Changing variables in $\int \mathrm{d} x_{0} / y_{0}$, the elliptic integral of the first kind, yields

$$
\int\left[\left(\frac{y_{0}}{\nu}\right)^{-1} \frac{\mathrm{d} x_{0}}{\mathrm{~d} B}\right] \frac{\mathrm{d} B}{\nu}=\int \frac{\mathrm{d} x_{0}}{y_{0}} .
$$

The quantity in braces, which is rational in $B$, is guaranteed to be a polynomial in $B$ of degree less than or equal to $g-1$, since the left-hand integrand is a pulledback version of the right-hand one, and must therefore be a holomorphic 1-form. Equation (5.1) supplies a linear constraint among the $g$ basic hyperelliptic integrals of the first kind. It reduces the number of independent integrals from $g$ to $g-1$.

The cases $\ell=2,3$ of (5.1) may be instructive. The maps $(B, \nu) \mapsto\left(x_{0}, y_{0}\right)$ were given in $(4.7),(4.9)$, and the degree- $(2 \ell+1)$ spectral polynomials $\mathrm{L}_{\ell}\left(\cdot ; g_{2}, g_{3}\right)$ follow from table 3 . If $\ell=2$, one obtains the hyperelliptic-to-elliptic reduction

$$
\int \frac{\left[\frac{3}{2} B\right] \mathrm{d} B}{\sqrt{\left(B^{2}-3 g_{2}\right)\left(B^{3}-\frac{9}{4} g_{2} B+\frac{27}{4} g_{3}\right)}}=\int \frac{\mathrm{d} x_{0}}{\sqrt{4 x_{0}^{3}-g_{2} x_{0}-g_{3}}}
$$


Table 7. Polynomials specifying the holomorphic 1-forms pulled back from $E_{g_{2}, g_{3}}$

\begin{tabular}{ll}
\hline$\ell$ & $\hat{P}_{\ell}\left(B ; g_{2}, g_{3}\right)$ \\
\hline 1 & 1 \\
2 & $B$ \\
3 & $B^{2}-\frac{15}{4} g_{2}$ \\
4 & $B^{3}-\frac{91}{4} g_{2} B+\frac{175}{2} g_{3}$ \\
5 & $B^{4}-\frac{321}{4} g_{2} B^{2}+\frac{2835}{4} g_{3} B+\frac{891}{2} g_{2}^{2}$ \\
6 & $B^{5}-\frac{861}{4} g_{2} B^{3}+\frac{12879}{4} g_{3} B^{2}+\frac{2425}{4} g_{2}^{2} B-\frac{280665}{4} g_{2} g_{3}$ \\
7 & $B^{6}-\frac{973}{2} g_{2} B^{4}+10813 g_{3} B^{3}+\frac{681373}{16} g_{2}^{2} B^{2}-\frac{2145143}{2} g_{2} g_{3} B+\frac{54071875}{16} g_{3}^{2}-\frac{5417685}{16} g_{2}^{3}$ \\
8 & $B^{7}-\frac{1953}{2} g_{2} B^{5}+29916 g_{3} B^{4}+\frac{3335445}{16} g_{2}^{2} B^{3}-\frac{34152435}{4} g_{2} g_{3} B^{2}$ \\
& $\quad+\left(-\frac{122490225}{16} g_{2}^{3}+\frac{937038375}{16} g_{3}^{2}\right) B+179425125 g_{2}^{2} g_{3}$ \\
\hline
\end{tabular}

where the change of variables is performed by (4.7). If $\ell=3$, one obtains

$$
\int \frac{\left[3\left(B^{2}-\frac{15}{4} g_{2}\right)\right] \mathrm{d} B}{\sqrt{\mathrm{L}_{3}\left(B ; g_{2}, g_{3}\right)}}=\int \frac{\mathrm{d} x_{0}}{\sqrt{4 x_{0}^{3}-g_{2} x_{0}-g_{3}}}
$$

where the full spectral polynomial $\mathrm{L}_{3}\left(B ; g_{2}, g_{3}\right)$ is

$$
B\left(B^{6}-\frac{63}{2} g_{2} B^{4}+\frac{297}{2} g_{3} B^{3}+\frac{4185}{16} g_{2}^{2} B^{2}-\frac{18225}{8} g_{2} g_{3} B-\frac{3375}{16} g_{2}^{3}+\frac{91125}{16} g_{3}^{2}\right)
$$

and the change of variables is performed by (4.9). These two reductions were known to Hermite (Königsberger 1878, Belokolos et al. 1986). More recently, the reductions induced by the $\ell=4,5$ coverings were worked out (Enol'skii \& Kostov 1994, Eilbeck $\&$ Enol'skii 1994). But the reductions with $\ell>5$ proved too difficult to compute.

We have now computed all reductions with $\ell \leq 8$, and Conjecture $\mathrm{L}$ makes possible the computation of reductions with much larger values of $\ell$. On the basis of results on $\ell \leq 8$, we make the following conjecture.

Conjecture 5.1. For all integer $\ell \geq 1$, the polynomial function $P_{\ell}\left(B ; g_{2}, g_{3}\right)$ in the hyperelliptic-to-elliptic reduction formula

$$
\int \frac{P_{\ell}\left(B ; g_{2}, g_{3}\right) \mathrm{d} B}{\sqrt{\mathrm{L}_{\ell}\left(B ; g_{2}, g_{3}\right)}}=\int \frac{\mathrm{d} x_{0}}{\sqrt{4 x_{0}^{3}-g_{2} x_{0}-g_{3}}},
$$

where the change of variables $x_{0}=x_{0}\left(B ; g_{2}, g_{3}\right)$ is given by Conjecture L, equals $\ell(\ell+1) / 4$ times a polynomial $\hat{P}_{\ell}\left(B ; g_{2}, g_{3}\right)$ which is monic and of degree $\ell-1$ in $B$.

The polynomials $\hat{P}_{\ell}$ are listed in Table $7 . \hat{P}_{4}, \hat{P}_{5}$ agree with those found by Enol'skii et al., if allowance is made for differences in normalization conventions.

A complete analysis of Lamé-derived elliptic covers will need to consider exceptional cases of several kinds. The covering curve $\Gamma_{\ell}\left(g_{2}, g_{3}\right)$ generically has genus $g=\ell$, but if the Klein invariant $J=g_{2}^{3} /\left(g_{2}^{3}-27 g_{3}^{2}\right)$ is a root of one of the two Cohn polynomials of table 4 , the genus will be reduced to $\ell-1$. According to conjecture 3.2 , this will happen, for instance, if $\ell \equiv 2(\bmod 3)$ and $g_{2}=0$ (i.e., $\left.J=0\right)$, so that the base curve $E_{g_{2}, g_{3}}$ is equianharmonic. When $g$ is reduced to $\ell-1$ in this way, the linear space of holomorphic 1-forms will be spanned by $B^{r-1}\left(B-B_{0}\right) \mathrm{d} B / \nu$, $r=1, \ldots, \ell-1$, where $B_{0}$ is the degenerate root of the spectral polynomial; but (5.1) will still provide a linear constraint on the associated hyperelliptic integrals.

Another sort of degeneracy takes place when the modular discriminant $\Delta \equiv$ $g_{2}^{3}-27 g_{3}^{2}$ equals zero, i.e., when $J=\infty$. In this case $E_{g_{2}, g_{3}}$ will degenerate to a 
rational curve, due to two or more of $e_{1}, e_{2}, e_{3}$ being coincident. The Lamé-derived reduction formulas, such as (5.2)-(5.3), continue to apply. (They are valid though trivial even in the case $e_{1}=e_{2}=e_{3}$, in which $g_{2}=g_{3}=0$.) So these formulas include as special cases certain hyperelliptic-to-rational reductions.

Subtle degeneracies of the covering map $\pi_{\ell}$ can occur, even in the generic case when $\Gamma_{\ell}$ has genus $\ell$ and $E_{g_{2}, g_{3}}$ has genus 1 . The branching structure of $\pi_{\ell}$ is determined by the polynomials $\hat{P}_{\ell}$ of table 7 , which are proportional to $\mathrm{d} x_{0} / \mathrm{d} B$. By examination, if $\hat{P}_{\ell}$ (conjectured to be of degree $\ell-1$ ) has distinct roots $\left\{B^{(i)}\right\}_{i=1}^{\ell-1}$, then $\pi_{\ell}$ will normally have $2 \ell-2$ simple critical points on $\Gamma_{\ell}$, namely the points $\left\{\left(B^{(i)}, \pm \nu^{(i)}\right)\right\}_{i=1}^{\ell-1}$. However, if any $B^{(i)}$ is located at a band edge, i.e., at a branch point of the hyperelliptic $(B, \nu)$-curve, then $\left(B^{(i)}, 0\right)$ will be a double critical point. This appears to happen whenever $\ell \equiv 0(\bmod 3)$ and the base curve $E_{g_{2}, g_{3}}$ is equianharmonic; the double critical point being located at $(B, \nu)=(0,0)$. Even if none of the roots of $\hat{P}_{\ell}$ is located at a band edge, it is possible for it to have a double root, in which case each of a pair of points $\left(B^{(i)}, \pm \nu^{(i)}\right)$ will be a double critical point. By examination, this happens when $\ell=4$ and $J=-2^{2} 5^{4} / 3^{5} 53$.

A few hyperelliptic-to-elliptic reductions, similar to the quadratic $(N=2)$ reduction of Legendre and Jacobi, can be found in handbooks of elliptic integrals (Byrd \& Friedman 1954, §575-§576). The Lamé-derived reductions, indexed by $\ell$, should certainly be included in any future handbook. It is natural to wonder whether they can be generalized in some straightforward way. The problem of finding the genus- 2 covers of an elliptic curve was intensively studied in the nineteenth century, by Weierstrass and Poincaré among many others, and one may reason by analogy with results on $\ell=2$. One expects that for all $\ell \geq 2$ and for arbitrarily large $N$, a generic $E_{g_{2}, g_{3}}$ can be covered by some genus- $\ell$ curve via a covering map of degree $N$. Each Lamé-derived covering $\pi_{\ell}: \Gamma_{\ell}\left(g_{2}, g_{3}\right) \rightarrow E_{g_{2}, g_{3}}$ has $N=\ell(\ell+1) / 2$ and may be only a low-lying member of an infinite sequence of coverings. Generalizing the Lamé-derived coverings may be possible even if one confines oneself to $N=\ell(\ell+1) / 2$. One can of course pre-compose with an automorphism of $\Gamma_{\ell}\left(g_{2}, g_{3}\right)$ and post-compose with an automorphism of $E_{g_{2}, g_{3}}$ (a modular transformation). But when $\ell=2$ a quite different covering map with the same degree is known to exist (Belokolos et al. 1986). $\pi_{2}$ has two simple critical points on $\Gamma_{2}$, but the other degree-3 covering map has a single double critical point on its analogue of $\Gamma_{2}$. Both can be generalized to include a free parameter (Burnside 1892, Belokolos $\&$ Enol'skii 2000). It seems possible that when $\ell>2$, similar alternatives to the Lamé-derived coverings may exist, with degree $\ell(\ell+1) / 2$ but different branching structures.

\section{Dispersion relations}

It is now possible to introduce dispersion relations, and determine the way in which the Hermite-Krichever Ansatz reduces higher- $\ell$ to $\ell=1$ dispersion relations. The starting point is the fundamental multi-valued function $\Phi$ introduced in $\S 2$. As noted, if the parametrization point $\left(x_{0}, y_{0}\right)$ on the punctured elliptic curve $E_{g_{2}, g_{3}} \backslash\{(\infty, \infty)\}$ is over $x_{0}=B \in \mathbb{C}$, then $\Phi\left(\cdot, \cdot ; x_{0}, y_{0}\right)$ will be a solution of the $\ell=1$ case of the Lamé equation (2.9). $E_{g_{2}, g_{3}}$ is defined by $y^{2}=4 x^{3}-g_{2} x-g_{3}$, so the hypothesis here is that $\left(x_{0}, y_{0}\right)$ should equal $\left(B, \pm \sqrt{4 B^{3}-g_{2} B-g_{3}}\right)$. 
In the Jacobi form (with independent variable $\alpha$ ), resp. the Weierstrassian form (with independent variable $u$ ), the crystal momentum $k$ characterizes the behavior of a solution of the Lamé equation under $\alpha \leftarrow \alpha+2 K$, resp. $u \leftarrow u+2 \omega$. Both shifts correspond to motion around $E_{g_{2}, g_{3}}$, along a fundamental loop that passes between $(x, y)=\left(e_{1}, 0\right)$ and $(\infty, \infty)$, and cannot be shrunk to a point. (If $e_{1}, e_{2}, e_{3}$ are defined by (2.1), this will be because $y$ is positive on one-half of the loop, and negative on the other.) By definition, the solution will be multiplied by $\xi \equiv \exp [\mathrm{i} k(2 K)]=$ $\exp [\mathrm{i} k(2 \omega)]$. It follows from the definition $(2.13)$ of $\Phi$ that when $\ell=1$,

$$
\xi=\exp [\mathrm{i} k(2 \omega)]=\exp \left[\frac{1}{2} \oint\left(\frac{y+y_{0}}{x-x_{0}}\right) \frac{\mathrm{d} x}{y}\right]
$$

That is, when $\ell=1$ the crystal momentum is given by a complete elliptic integral.

It was pointed out in $\S 4$ that the spectral curve $\Gamma_{1}$ that parametrizes $\ell=1$ solutions can be identified with $E_{g_{2}, g_{3}}$ itself, via the identification $(B, \nu)=\left(x_{0}, y_{0} / 2\right)$. This suggests a subtle but important reinterpretation of $k$. Until now we have regarded it as a function of the energy parameter, here $B$, which is determined only up to integer multiples of $\pi / K=\pi / \omega$, and which is also undetermined as to sign. If the presence of $y_{0}=2 \nu$ in (6.1) is taken into account, it is clear that the $\ell=1$ crystal momentum, which we call $k_{1}$ henceforth, should be regarded as a function not on $\mathbb{P}^{1} \backslash\{\infty\} \ni B$, but rather on $\Gamma_{1} \backslash\{(\infty, \infty)\} \ni(B, \nu)$. In this interpretation, the indeterminacy of sign disappears. The additive indeterminacy, on account of which $k_{1}$ is an elliptic function of the second kind, remains but can be viewed as an artifact: it is due to $k_{1} \propto \log \xi$, where $\xi$ is the Floquet multiplier. The behavior of $k_{1}$ near the puncture $(B, \nu)=(\infty, \infty)$ is easily determined. It follows from (6.1) that as $(B, \nu) \rightarrow(\infty, \infty)$, i.e., $\left(x_{0}, y_{0}\right) \rightarrow(\infty, \infty)$, each branch of $k_{1}$ is asymptotic to $-\mathrm{i} \nu / B$ to leading order. Since $B=x_{0}, \nu=2 y_{0}$ have double and triple poles there, respectively, it follows that each branch of $k_{1}$ has a simple pole at the puncture.

The multiplier $\xi$ is a true single-valued function on the punctured spectral curve $\Gamma_{1} \backslash\{(\infty, \infty)\}$, and moreover is entire. We can write $\xi: \Gamma_{1} \backslash\{(\infty, \infty)\} \rightarrow \mathbb{P}^{1} \backslash\{0, \infty\}$, since the multiplier is never zero. We remark that like $k_{1}$, this function is not algebraic: it necessarily has an essential singularity at the puncture. The $((B, \nu), \xi)-$ curve over $\Gamma_{1} \backslash\{(\infty, \infty)\} \ni(B, \nu)$, which is a single cover, and the $(B, \xi)$-curve over $\mathbb{C} \ni B$, which is a double cover, are both transcendental curves.

Let us tentatively regard the crystal momentum for each integer $\ell \geq 1$ as an additively multi-valued function on the punctured spectral curve $\Gamma_{\ell} \backslash\{(\infty, \infty)\}$. We shall write it as $k_{\ell}\left(B, \nu ; g_{2}, g_{3}\right)$, with the understanding that for this to be welldefined, $B, \nu$ must be related by the spectral curve relation $\nu^{2}=\mathrm{L}_{\ell}\left(B ; g_{2}, g_{3}\right)$. The quantity $k_{\ell}\left(B, \nu ; g_{2}, g_{3}\right)$ will not be undetermined as to sign. Let us also regard the projections $\pi_{\ell}: \Gamma_{\ell} \rightarrow E_{g_{2}, g_{3}}$ of the Hermite-Krichever Ansatz as maps $\pi_{\ell}: \Gamma_{\ell} \rightarrow \Gamma_{1}$. That is, $\pi_{\ell}$ maps $(B, \nu) \in \Gamma_{\ell}$ to the point $\left(B^{\prime}, \nu^{\prime}\right) \equiv\left(x_{0}, y_{0} / 2\right) \in \Gamma_{1}$. The reductions $(B, \nu) \mapsto\left(B^{\prime}, \nu^{\prime}\right)$ for $\ell=2,3$, for example, follow from (4.7)-(4.10).

Proposition 6.1. Suppose that the integration of the Lamé equation on the elliptic curve $E_{g_{2}, g_{3}}$, for integer $\ell \geq 1$, can be accomplished in the framework of the Hermite-Krichever Ansatz by maps $\pi_{\ell}: \Gamma_{\ell} \rightarrow \Gamma_{1}$ and $\kappa_{\ell}: \Gamma_{\ell} \rightarrow \mathbb{P}^{1}$, where $\pi_{\ell}$ and $\kappa_{\ell}$ map the point $(B, \nu)$ to $\left(B_{\ell}\left(B ; g_{2}, g_{3}\right), \nu_{\ell}\left(B, \nu ; g_{2}, g_{3}\right)\right)$ and $\hat{\kappa}_{\ell}\left(B ; g_{2}, g_{3}\right) \nu$, respectively. Then the dispersion relation for the Hermite-Krichever solutions will 
be $k=k_{\ell}\left(B, \nu ; g_{2}, g_{3}\right)$, in which $k_{\ell}$ can be expressed in terms of $k_{1}$ by

$$
k_{\ell}\left(B, \nu ; g_{2}, g_{3}\right)=k_{1}\left(B_{\ell}\left(B, \nu ; g_{2}, g_{3}\right), \nu_{\ell}\left(B, \nu ; g_{2}, g_{3}\right)\right)-\mathrm{i} \hat{\kappa}_{\ell}\left(B, \nu ; g_{2}, g_{3}\right) \nu
$$

This proposition follows immediately from the form of the Hermite-Krichever solutions (4.1). The first term in (6.2) arises from the $\Phi, \Phi^{\prime}$ factors, and the second from the exponential. The factors $\mathcal{A}, \mathcal{B}$ do not contribute to the crystal momentum.

The effort expended in replacing two-valuedness by single-valuedness is justified by the following observation. As a function of $B$ alone, rather than of the pair $(B, \nu)$, each of the two terms of (6.2) would be undetermined as to sign. This ambiguity could cause confusion and errors. The present formulation, though a bit pedantic, facilitates the determination of the correct relative sign.

The form of the $(B, \nu) \mapsto\left(B^{\prime}, \nu^{\prime}\right)$ map assumed in the proposition is of course the form supplied by Conjecture L. Substituting the formulas of the conjecture into (6.2) yields an explicit expression for $k_{\ell}$. It follows readily from this expression that for all integer $\ell \geq 1$, each branch of $k_{\ell}$ on the hyperelliptic $(B, \nu)$-curve $\Gamma_{\ell}\left(g_{2}, g_{3}\right)$ satisfies $k_{\ell} \sim-\mathrm{i} \nu / B^{\ell},(B, \nu) \rightarrow(\infty, \infty)$. Since $B, \nu$ have double and order- $(2 \ell+1)$ poles at the puncture $(\infty, \infty)$, respectively, this implies that irrespective of $\ell$, each branch of the crystal momentum has a simple pole at the puncture.

\section{Band structure of the Jacobi form}

The results of $\S 3-\S 6$ were framed in terms of the elliptic-curve algebraic form of the Lamé equation. Most work on Lamé dispersion relations has used the Jacobi form instead, and has led accordingly to expressions involving Jacobi theta functions. To derive dispersion relations that can be compared with previous work, the formulation of $\S 6$ must be converted to the language of the Jacobi form.

The relationships among the several forms were sketched in $\S 2$. In the Weierstrassian and Jacobi forms, the Lamé equation is an equation on $\mathbb{C}$ with doubly periodic coefficients, rather than an equation on the curve $E_{g_{2}, g_{3}}$. In the conversion to the Jacobi form the invariants $g_{2}, g_{3}$ are expressed in terms of the modular parameter $m$ by $(2.2)$, with $A=1$ by convention. The coordinate $x$ on $E_{g_{2}, g_{3}}$ is interpreted as the function $\wp\left(u ; g_{2}, g_{3}\right)$, i.e., as $m \operatorname{sn}^{2}(\alpha \mid m)-\frac{1}{3}(m+1)$, where $u \in \mathbb{C}$ and $\alpha \equiv u-\mathrm{iK}^{\prime}(m) \in \mathbb{C}$ are the respective independent variables of the Weierstrassian and Jacobi forms. The holomorphic differential $\mathrm{d} x / y$ corresponds to $\mathrm{d} u$ or $\mathrm{d} \alpha$, and the derivative $y \mathrm{~d} / \mathrm{d} x$ to $\mathrm{d} / \mathrm{d} u$ or $\mathrm{d} / \mathrm{d} \alpha$. The coordinate $y=(y \mathrm{~d} / \mathrm{d} x) x$ on $E_{g_{2}, g_{3}}$ is interpreted as $\wp^{\prime}\left(u ; g_{2}, g_{3}\right)$, i.e., as the doubly periodic function $2 m \operatorname{sn}(\alpha \mid m) \operatorname{cn}(\alpha \mid m) \operatorname{dn}(\alpha \mid m)$ on the complex $\alpha$-plane. The functions $\sqrt{x-e_{\gamma}}, \gamma=1,2,3$, correspond to $-\mathrm{i} \operatorname{dn}(\alpha \mid m),-\mathrm{i} m^{1 / 2} \operatorname{cn}(\alpha \mid m)$ and $m^{1 / 2} \operatorname{sn}(\alpha \mid m)$.

$$
B=-E+\frac{1}{3} \ell(\ell+1)(m+1)
$$

relates the accessory parameters $B, E$ of the different forms.

With these formulas, it is easy to convert the Lamé polynomials of table 2 to polynomials in $\operatorname{sn}(\alpha \mid m), \operatorname{cn}(\alpha \mid m), \operatorname{dn}(\alpha \mid m)$, and the spectral polynomials of table 3 to polynomials in $E$, for comparison with the list given by Arscott $(1964, \S 9.3 .2)$.

Definition. The Jacobi-form spectral polynomial $\widetilde{\mathrm{L}}_{\ell}(E \mid m)$ is the negative of the spectral polynomial $\mathrm{L}_{\ell}\left(B ; g_{2}, g_{3}\right)$, when $B ; g_{2}, g_{3}$ are expressed in terms of $E, m$. 
It is a monic degree- $(2 \ell+1)$ polynomial in $E$ with coefficients polynomial in $m$, and can be regarded as $\prod_{s=1}^{2 \ell+1}\left[E-E_{s}(m)\right]$, where the roots $\left\{E_{s}\right\}$ are the values of the energy $E$ for which there exists a Lamé polynomial solution of the Lamé equation, counted with multiplicity. (The negation is due to the relative minus sign in the $B \leftrightarrow E$ correspondence (7.1).)

Definition. The $\ell$ 'th Jacobi-form spectral curve $\widetilde{\Gamma}_{\ell} \equiv \widetilde{\Gamma}_{\ell}(m)$ is the hyperelliptic curve over $\mathbb{P}^{1} \ni E$ comprising all $(E, \tilde{\nu})$ satisfying $\tilde{\nu}^{2}=\widetilde{\mathrm{L}}_{\ell}(E \mid m)$. In the usual case when $m$ is real, $\tilde{\nu}$ will be real if $E$ is in a band, and non-real if $E$ is in a lacuna. In both cases it is determined only up to negation. By convention, the correspondence between the curve $\widetilde{\Gamma}_{\ell} \ni(E, \tilde{\nu})$ and the previously introduced curve $\Gamma_{\ell} \ni(B, \nu)$, which was defined by $\nu^{2}=\mathrm{L}_{\ell}\left(B ; g_{2}, g_{3}\right)$, is given by $\nu=+\mathrm{i} \tilde{\nu}$.

The following cases are examples. When $\ell=1,2,3$, the spectral polynomial factors over the integers into polynomials at most quadratic in $E$.

$$
\begin{aligned}
\widetilde{\mathrm{L}}_{1}(E \mid m)= & (E-1)(E-m)(E-m-1) \\
\widetilde{\mathrm{L}}_{2}(E \mid m)= & {\left[E^{2}-4(m+1) E+12 m\right](E-m-1)(E-4 m-1)(E-m-4) } \\
\widetilde{\mathrm{L}}_{3}(E \mid m)=( & E-4 m-4)\left[E^{2}-2(2 m+5) E+3(8 m+3)\right] \\
& \times\left[E^{2}-2(5 m+2) E+3\left(3 m^{2}+8 m\right)\right] \\
& \times\left[E^{2}-10(m+1) E+3\left(3 m^{2}+26 m+3\right)\right] .
\end{aligned}
$$

In (7.3) and (7.4) the first factor arises from $L_{\ell}^{\mathrm{I}}\left(B ; g_{2}, g_{3}\right)$ and the remaining three from the factors $L_{\ell}^{\mathrm{II}}\left(B ; e_{\gamma}, g_{2}, g_{3}\right), \gamma=1,2,3$. In (7.2) there is no Type I factor.

Dispersion relations in their Jacobi form can now be investigated. Recall that if $\ell=1$, the Jacobi-form Lamé equation (1.1) has $\widetilde{\Phi}\left(\cdot ; \alpha_{0} \mid m\right)$ as a solution, where the theta quotient $\widetilde{\Phi}$ (the Jacobi-form version of Halphen's l'élément simple) is defined in (1.3), and the multi-valued parameter $\alpha_{0}$ is defined by $\operatorname{dn}^{2}\left(\alpha_{0} \mid m\right)=E-m$. This solution has crystal momentum $k=k_{1}$ equal to $-\mathrm{iZ}\left(\alpha_{0} \mid m\right)+\pi / 2 \mathrm{~K}(m)$, which is undetermined up to sign, and is also determined only up to integer multiples of $\pi / \mathrm{K}(m)$. The sign indeterminacy is due to $\operatorname{dn}^{2}(\cdot \mid m)$ being even. This causes $\alpha_{0}$ to be undetermined up to sign, and $k_{1}$ as well, since the function $\mathrm{Z}(\cdot \mid m)$ is odd.

The parametrization point $\alpha_{0}$, or the equivalent point $u_{0} \equiv \alpha_{0}+\mathrm{iK}^{\prime}(m)$ of the Weierstrassian form, corresponds to the parametrization point $\left(x_{0}, y_{0}\right)$ of the fundamental function $\Phi$ on the elliptic curve $E_{g_{2}, g_{3}}$. The correspondence is the usual one: $x_{0}=\wp\left(u_{0} ; g_{2}, g_{3}\right), y_{0}=\wp^{\prime}\left(u_{0}, g_{2}, g_{3}\right)$. The first of these two equations says that $x_{0}=$ $m \operatorname{sn}^{2}\left(\alpha_{0} \mid m\right)-\frac{1}{3}(m+1)$, and the latter that $y_{0}=2 m \operatorname{sn}\left(\alpha_{0} \mid m\right) \operatorname{cn}\left(\alpha_{0} \mid m\right) \operatorname{dn}\left(\alpha_{0} \mid m\right)$. The formula which computes $\alpha_{0}$ from $E$, namely $\operatorname{dn}^{2}\left(\alpha_{0} \mid m\right)=E-m$, is readily seen to be a translation to the Jacobi form of the familiar condition $x_{0}=B$, which simply says that the parametrization point $\left(x_{0}, y_{0}\right)$ must be 'over' $B \in \mathbb{C}$.

The correspondence between the Jacobi and elliptic-curve forms motivates the following reinterpretation of the crystal momentum of the fundamental solution $\widetilde{\Phi}$, which is modelled on the reinterpretation of the last section. $k_{1}$ should be viewed as a function not of the energy $E \in \mathbb{C}$, but rather of a point $(E, \tilde{\nu})$ on the punctured Jacobi-form spectral curve $\widetilde{\Gamma}_{1}(m) \backslash\{(\infty, \infty)\}$. There are two such points for each energy $E$, except when $E$ is a band edge. This is the source of the sign ambiguity 
in the parameter $\alpha_{0}$. Since $y_{0}=2 \nu=2 \mathrm{i} \tilde{\nu}$, the equation

$$
m \operatorname{sn}\left(\alpha_{0} \mid m\right) \operatorname{cn}\left(\alpha_{0} \mid m\right) \operatorname{dn}\left(\alpha_{0} \mid m\right)=\mathrm{i} \tilde{\nu}
$$

determines a unique sign for $\alpha_{0}$, provided that $\tilde{\nu}$ is specified in addition to $E$. We shall write $k_{1}$ as $k_{1}(E, \tilde{\nu} \mid m)$, with the understanding that for this to be welldefined, the pair $E, \tilde{\nu}$ must be related by the spectral curve relation $\tilde{\nu}^{2}=\widetilde{L}_{\ell}(E \mid m)$. The additively undetermined quantity $k_{1}(E, \tilde{\nu} \mid m)$ will not be undetermined as to sign. It is easily checked that on each branch, $k_{1} \sim \tilde{\nu} / E$ as $(E, \tilde{\nu}) \rightarrow(\infty, \infty)$.

Definition. A solution of the Jacobi-form Lamé equation (1.1) is said to be an Hermite-Krichever solution if it is of the form

$$
\left[\widetilde{\mathcal{A}}\left(\operatorname{sn}^{2}(\alpha \mid m)\right) \widetilde{\Phi}\left(\alpha ; \alpha_{0} \mid m\right)+2 \widetilde{\mathcal{B}}\left(\operatorname{sn}^{2}(\alpha \mid m)\right) \widetilde{\Phi}^{\prime}\left(\alpha ; \alpha_{0} \mid m\right)\right] \exp (\kappa \alpha),
$$

for some $\alpha_{0} \in \mathbb{C}$ and $\kappa \in \mathbb{C}$. Here $\widetilde{\mathcal{A}}, \widetilde{\mathcal{B}}$ are polynomials, and $\widetilde{\Phi}^{\prime} \equiv(\mathrm{d} / \mathrm{d} \alpha) \widetilde{\Phi}$.

The expression (7.6) is a replacement for the original Jacobi-form expression (1.5), to which it is equivalent. Regardless of which is used, it is easy to compute the crystal momentum of an Hermite-Krichever solution. The momentum computed from (7.6) will be $\left[-\mathrm{i} \mathrm{Z}\left(\alpha_{0} \mid m\right)+\pi / 2 \mathrm{~K}(m)\right]-\mathrm{i} \kappa$, up to additive multi-valuedness. The first term arises from the $\widetilde{\Phi}, \widetilde{\Phi}^{\prime}$ factors, and the second from the exponential. The factors $\widetilde{\mathcal{A}}, \widetilde{\mathcal{B}}$ do not contribute, since $\operatorname{sn}^{2}(\alpha \mid m)$ is periodic in $\alpha$ with period $2 \mathrm{~K}(m)$.

The Jacobi form of the Hermite-Krichever Ansatz asserts that for all integer $\ell$ and $m \in \mathbb{C} \backslash\{0,1\}$, there is an Hermite-Krichever solution for all but a finite number of values of the energy $E$. On the elliptic curve $E_{g_{2}, g_{3}}$, these solutions were constructed from two maps: a projection $\pi_{\ell}: \Gamma_{\ell} \rightarrow E_{g_{2}, g_{3}}$ and an auxiliary function $\kappa: \Gamma_{\ell} \rightarrow \mathbb{P}^{1}$. But $\pi_{\ell}$ should really be regarded as a map from $\Gamma_{\ell}$ to $\Gamma_{1}$, on account of the correspondence between $E_{g_{2}, g_{3}}$ and $\Gamma_{1}$ provided by $\left(x_{0}, y_{0}\right)=(B, 2 \nu)$. The following is the Jacobi-form version of proposition 6.1.

Proposition 7.1. Suppose that the integration of the Lamé equation on the elliptic curve $E_{g_{2}, g_{3}}$, for integer $\ell \geq 1$, can be accomplished in the framework of the Hermite-Krichever Ansatz by the maps $\pi_{\ell}: \Gamma_{\ell} \rightarrow \Gamma_{1}$ and $\kappa_{\ell}: \Gamma_{\ell} \rightarrow \mathbb{P}^{1}$, where $\pi_{\ell}$ and $\kappa_{\ell}$ map the point $(B, \nu)$ to $\left(B_{\ell}\left(B ; g_{2}, g_{3}\right), \nu_{\ell}\left(B, \nu ; g_{2}, g_{3}\right)\right)$ and $\hat{\kappa}_{\ell}\left(B ; g_{2}, g_{3}\right) \nu$, respectively. Then the dispersion relation for the solutions of the Jacobi form of the Lamé equation will be $k=k_{\ell}(E, \tilde{\nu} \mid m)$, where up to additive multi-valuedness

$$
k_{\ell}(E, \tilde{\nu} \mid m)=k_{1}\left(\mathcal{E}_{\ell}(E \mid m), \tilde{\nu}_{\ell}(E, \tilde{\nu} \mid m) \mid m\right)+\hat{\kappa}_{\ell}(E \mid m) \tilde{\nu}
$$

in which

$$
\begin{aligned}
\mathcal{E}_{\ell}(E \mid m) & \equiv-B_{\ell}\left(-E+\frac{1}{3} \ell(\ell+1)(m+1) ; g_{2}(m), g_{3}(m)\right)+\frac{2}{3}(m+1), \\
\tilde{\nu}_{\ell}(E, \tilde{\nu} \mid m) & \equiv-\mathrm{i} \nu_{\ell}\left(-E+\frac{1}{3} \ell(\ell+1)(m+1), \mathrm{i} \tilde{\nu} ; g_{2}(m), g_{3}(m)\right), \\
\hat{\kappa}_{\ell}(E \mid m) & \equiv \hat{\kappa}_{\ell}\left(-E+\frac{1}{3} \ell(\ell+1)(m+1) ; g_{2}(m), g_{3}(m)\right) .
\end{aligned}
$$

The formula (7.7) follows by inspection. The projection $\pi_{\ell}$ reduces the integration of the Lamé equation to the integration of an $\ell=1$ equation, the ' $B$ ' parameter of which equals $B_{\ell}\left(B ; g_{2}, g_{3}\right)$. By (7.1), the ' $E$ ' parameter of the $\ell=1$ equation will 

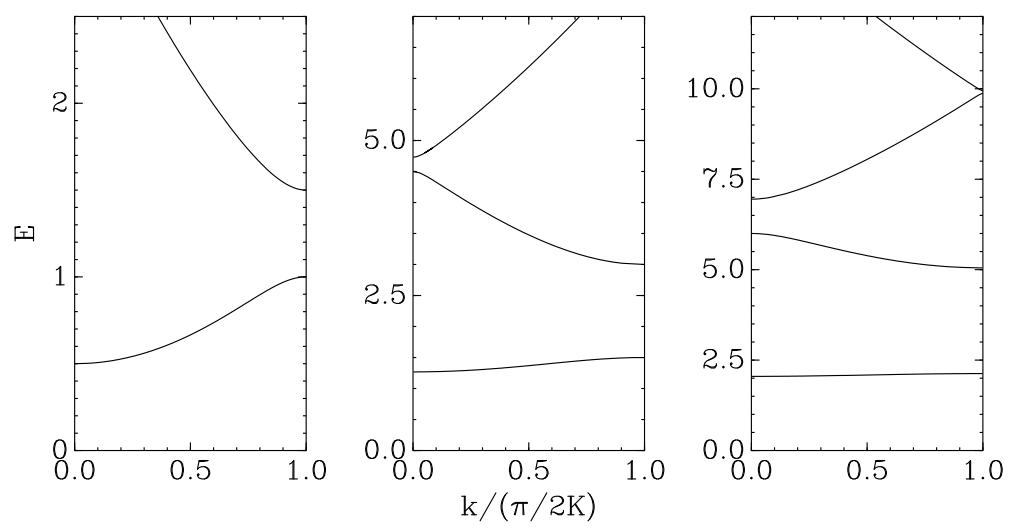

Figure 1. Dispersion relations for $\ell=1,2,3$ in the lemniscatic case $m=\frac{1}{2}$.

be the right-hand side of (7.8). The two terms of (7.7) are simply the two terms of $\left[-\mathrm{i} \mathrm{Z}\left(\alpha_{0} \mid m\right)+\pi / 2 \mathrm{~K}(m)\right]-\mathrm{i} \kappa$. The equality $\nu=\mathrm{i} \tilde{\nu}$ has been used.

It is straightforward to apply proposition 7.1 to the cases $\ell=2,3$, since the coverings $\pi_{2}, \pi_{3}$ and auxiliary functions $\kappa_{2}, \kappa_{3}$ were worked out in $\S 4$. A brief discussion of the $\ell=2$ case should suffice. After some algebra, we find

$$
\begin{aligned}
\mathcal{E}_{2}(E \mid m) & =\frac{E^{3}-12(m+1)^{2} E-4(m+1)\left(4 m^{2}-19 m+4\right)}{9\left[E^{2}-4(m+1) E+12 m\right]}, \\
\tilde{\nu}_{2}(E, \tilde{\nu} \mid m) & =-\frac{(E+2 m-4)(E-4 m+2)(E-4 m-4)}{27\left[E^{2}-4(m+1) E+12 m\right]^{2}} \tilde{\nu} \\
\hat{\kappa}_{2}(E \mid m) & =-\frac{2}{3\left[E^{2}-4(m+1) E+12 m\right]}
\end{aligned}
$$

from which $k_{2}(E, \tilde{\nu} \mid m)$ may be computed by (7.7). Like $k_{1}, k_{2}$ is determined only up to integer multiples of $\pi / K \equiv \pi / \mathrm{K}(m)$. Each branch of $k_{2}$ has the property that $k_{2}(E, \tilde{\nu} \mid m) \sim \pm E^{1 / 2}, E \rightarrow+\infty$, with ' \pm ' determined by the sign of $\tilde{\nu}=\tilde{\nu}(E)$. This is a special case of a general fact: for all integer $\ell \geq 1, k_{\ell}(E, \tilde{\nu} \mid m) \sim \pm E^{1 / 2}$, $E \rightarrow+\infty$, since each branch of $k_{\ell}$ is asymptotic to $(-)^{\ell-1} \tilde{\nu} / E^{\ell}$ as $(E, \tilde{\nu}) \rightarrow(\infty, \infty)$.

The real portions of the dispersion relations $k=k_{1}\left(E, \tilde{\nu} \mid \frac{1}{2}\right), k_{2}\left(E, \tilde{\nu} \mid \frac{1}{2}\right)$ and $k_{3}\left(E, \tilde{\nu} \mid \frac{1}{2}\right)$ are graphed in figure 1 . For ease of viewing, each crystal momentum is regarded as lying in the interval $[0, \pi / 2 K]$; which is equivalent to choosing the sign of $\tilde{\nu}=\tilde{\nu}(E)$ in an $E$-dependent way. As (7.2)-(7.4) imply, the two $\ell=1$ bands are $\left[\frac{1}{2}, 1\right],\left[\frac{3}{2}, \infty\right)$, the three $\ell=2$ bands are $\left[3-\sqrt{3}, \frac{3}{2}\right],\left[3, \frac{9}{2}\right],[3+\sqrt{3}, \infty)$, and the four $\ell=3$ bands are $\left[\frac{9}{2}-\sqrt{6}, 6-\sqrt{15}\right],\left[\frac{15}{2}-\sqrt{6}, 6\right],\left[\frac{9}{2}+\sqrt{6}, 6+\sqrt{15}\right],\left[\frac{15}{2}+\sqrt{6}, \infty\right)$.

Our $\ell=1$ graph agrees with that of Li et al. (2000, figure 6), and for confirmation, with that of Sutherland (1973, figure 1). Unfortunately, our $\ell=2$ graph disagrees with that of $\mathrm{Li}$ et al. in the placement or direction of curvature of each of the two upper bands. The algorithm they used for reducing $\ell=2$ to $\ell=1$, which was based on Hermite's solution of the Jacobi-form Lamé equation (Whittaker \& Watson 1927, §23.71), evidently yielded incorrect results for these bands. It appears that for the middle band, at least, the discrepancy can be traced to an incorrect choice of relative sign for the two terms of $k=k_{2}$. Our reinterpretation of the 
crystal momentum as a function on the spectral curve, rather than a function of the energy, eliminates such sign ambiguities.

\section{Conclusions}

In this paper we have developed a novel approach to the Hermite-Krichever Ansatz, applied to the Lamé equation. Conjecture L provides a formula for the covering map of the Ansatz, in terms of certain spectral polynomials which are of independent interest. The conjecture permits an efficient computation of Lamé dispersion relations, of a mixed symbolic-numerical kind. It has been verified for integer $\ell$ up to $\ell=8$, and its rigorous proof for all integer $\ell \geq 1$ should be a priority.

Our approach can be extended from the Lamé equation to the Heun equation, which as a differential equation on the elliptic curve $E_{g_{2}, g_{3}}$ has four regular singular points rather than one. Its Weierstrassian form is called the Treibich-Verdier equation (Smirnov 2002) and its Jacobi form the associated Lamé equation (Magnus \& Winkler 1979, §7.3). Applying the Ansatz to the Heun equation leads to a greater variety of coverings of $E_{g_{2}, g_{3}}$; for example, coverings by a genus- 2 hyperelliptic curve that have degrees 3, 4, 5 (Belokolos \& Enol'skii 2000). These play a role in the construction of elliptic soliton solutions of certain nonlinear evolution equations that arise in fibre optics (Christiansen et al. 2000). The extension of Conjecture L to the Heun equation will be considered elsewhere.

This work was partially supported by NSF grant PHY-0099484.

\section{References}

Abramowitz, M. \& Stegun, I. A. (eds) 1965 Handbook of mathematical functions. New York: Dover.

Arscott, F. M. 1964 Periodic differential equations. New York: Macmillan.

Arscott, F. M. \& Khabaza, I. M. 1962 Tables of Lamé polynomials, vol. 17 of Mathematical Tables Series. Oxford: Pergamon.

Belokolos, E. D. \& Enol'skii, V. Z. 2000 Reductions of Abelian functions and algebraically integrable systems, II. Itogi Nauki i Tekhniki: Seriya Sovrem. Mat. 75. (Transl. J. Math. Sci. (New York) 108, 295-374 (2002).)

Belokolos, E. D., Bobenko, A. I., Matveev, V. B. \& Enol'skii, V. Z. 1986 Algebraic-geometric principles of superposition of finite-zone solutions of integrable non-linear equations. Uspekhi Mat. Nauk 41(2), 3-42. (Transl. Russian Math. Surveys 41(2), 1-49 (1986).)

Boyanovsky, D., de Vega, H. J., Holman, R. \& Salgado, J. F. J. 1996 Analytic and numerical study of preheating dynamics. Phys. Rev. D 54, 7570-7598. Available as arXiv:hep$\mathrm{ph} / 9608205$.

Brezhnev, Y. V. 2000 Elliptic solitons and Gröbner bases. Preprint, available as arXiv:nlin.SI/0007028.

Burnside, W. 1892 On the form of hyperelliptic integrals of the first order which are expressible as the sum of two elliptic integrals. Proc. London Math. Soc. 23, 173-185.

Byrd, P. F. \& Friedman, M. D. 1954 Handbook of elliptic integrals for engineers and physicists. Springer-Verlag.

Caputo, J.-G., Flytzanis, N., Gaididei, Y., Stefanakis, N. \& Vavalis, E. 2000 Stability analysis of static solutions in a Josephson junction. Supercond. Sci. Technol. 13, 423-438. Available as arXiv:cond-mat/0010335. 
Christiansen, P. L., Eilbeck, J. C., Enol'skii, V. Z. \& Kostov, N. A. 2000 Quasi-periodic and periodic solutions for coupled nonlinear Schrödinger equations of Manakov type. Proc. R. Soc. Lond. A 456, 2263-2281. Available as arXiv:solv-int/9904017.

Chudnovksy, D. V. \& Chudnovsky, G. V. 1980 Remark on the nature of the spectrum of the Lamé equation. A problem from transcendence theory. Lett. Nuovo Cimento 29, $545-550$.

Cohn, F. 1888 Über Lamésche Funktionen mit komplexen Parametern. Ph.D. dissertation, University of Königsberg, Germany.

Dobner, H.-J. \& Ritter, S. 1998 Reliable computation of eigenvalues of the magnetostatic integral operator. Math. Comput. Modelling 27, 1-10.

Eilbeck, J. C. \& Enol'skii, V. Z. 1994 Elliptic Baker-Akhiezer functions and an application to an integrable dynamical system. J. Math. Phys. 35, 1192-1201.

Enol'skii, V. Z. \& Kostov, N. A. 1994 On the geometry of elliptic solitons. Acta Appl. Math. 36, $57-86$.

Erdélyi, A. (ed.) 1953-55 Higher transcendental functions. New York: McGraw-Hill.

Fernández C., D. J., Negro, J. \& Nieto, L. M. 2000 Second-order supersymmetric periodic potentials. Phys. Lett. 275A, 338-349.

Finkel, F., González-López, A. \& Rodríguez, M. A. 2000 A new algebraization of the Lamé equation. J. Phys. A 33, 1519-1542. Available as arXiv:math-ph/9908002.

Gerdt, V. P. \& Kostov, N. A. 1989 Computer algebra in the theory of ordinary differential equations of Halphen type. In Computers and mathematics (eds E. Kaltofen \& S. M. Watt), pp. 279-288. New York/Berlin: Springer-Verlag.

Gesztesy, F. \& Holden, H. 2003 Soliton equations and their algebro-geometric solutions, vol. I. Cambridge, UK: Cambridge University Press.

Grecchi, V. \& Sacchetti, A. 1997 Lifetime of the Wannier-Stark resonances and perturbation theory. Commun. Math. Phys. 185, 359-378.

Greene, P. B., Kofman, L., Linde, A. \& Starobinsky, A. A. 1997 Structure of resonance in preheating after inflation. Phys. Rev. D 56, 6175-6192. Available as arXiv:hep$\mathrm{ph} / 9705347$.

Guerritore, G. 1909 Calcolo delle funzioni di Lamè fino a quelle di grado 10. Giorn. Mat. Battaglini 47, 164-172.

Halphen, G.-H. 1888 Traité des fonctions elliptiques et de leurs applications, vol. II. Paris: Gauthier-Villars.

Ivanov, P. 2001 On Lamé's equation of a particular kind. J. Phys. A 34, 8145-8150. Available as arXiv:math-ph/0008008.

Kaiser, D. I. 1998 Resonance structures for preheating with massless fields. Phys. Rev. D 57, 702-711. Available as arXiv:hep-ph/9707516.

Kantowski, R. \& Thomas, R. C. 2001 Distance-redshift in inhomogeneous $\Omega_{0}=1$ Friedmann-Lemaître-Robertson-Walker cosmology. Astrophys. J. 561, 491-495. Available as arXiv:astro-ph/0011176.

Klein, F. 1892 Ueber den Hermite'schen Fall der Lamé'schen Differentialgleichungen. Math. Ann. 40, 123-129.

Königsberger, L. 1878 Ueber die Reduction hyperelliptischer Integrale auf elliptische. J. Reine Angew. Math. 85, 273-294.

Kostov, N. A. \& Enol'skii, V. Z. 1993 Spectral characteristics of elliptic solitons. Mat. Zametki 53(3), 62-71. (Transl. Math. Notes 53(3-4), 287-293 (1993).)

Krichever, I. M. 1980 Elliptic solutions of the Kadomtsev-Petviashvili equation and integrable systems of particles. Funktsional. Anal. i Prilozhen 14(4), 45-54, 95. (Transl. Funct. Anal. Appl. 14, 282-290 (1980).)

Krichever, I. M. 1990 Generalized elliptic genera and Baker-Akhiezer functions. Mat. Zametki 47(2), 34-45, 158. (Transl. Math. Notes 47(1-2), 132-142 (1990).) 
Li, H. \& Kusnezov, D. 1999 Group theory approach to band structure: Scarf and Lamé Hamiltonians. Phys. Rev. Lett. 83, 1283-1286. Available as arXiv:cond-mat/9907202.

Li, H., Kusnezov, D. \& Iachello, F. 2000 Group-theoretical properties and band structure of the Lamé Hamiltonian. J. Phys. A 33, 6413-6429.

Magnus, W. \& Winkler, S. 1979 Hill's equation, revised edn. New York: Dover.

Maier, R. S. 2002 Algebraic solutions of the Lamé equation, revisited. J. Differential Equations, to appear. Available as arXiv:math.CA/0206285.

Maier, R. S. \& Stein, D. L. 2001 Droplet nucleation and domain wall motion in a bounded interval. Phys. Rev. Lett. 87, 270601-1-270601-4. Available as arXiv:cond-mat/0108217.

McKean, H. P. \& van Moerbeke, P. 1979 The spectrum of Hill's equation. Invent. Math. 30, 217-274.

Sacchetti, A. 1997 Band functions for the Lamé equation. MapleTech 4(1), 28-33.

Smirnov, A. O. 2002 Elliptic solitons and Heun's equation. In The Kowalevski Property, CRM Proc. Lecture Notes, no. 32, pp. 287-305. Providence, RI: American Mathematical Society. Available as arXiv:math.CA/0109149.

Strutt, M. J. O. 1967 Lamésche- Mathieusche- und verwandte Funktionen in Physik und Technik. New York: Chelsea Publishing Co. Reprint of the 1932 edition.

Sutherland, B. 1973 Some exact results for one-dimensional models of solids. Phys. Rev. A 8, 2514-2516.

Turbiner, A. V. 1989 Lamé equation, sl(2) algebra and isospectral deformations. J. Phys. A 22, L1-L3.

van der Waall, A. 2002 Lamé equations with finite monodromy. Ph.D. dissertation, University of Utrecht, The Netherlands. Currently available at http://www.library.uu.nl/digiarchief/dip/diss/2002-0530-113355/inhoud.htm

Whittaker, E. T. \& Watson, G. N. 1927 A course of modern analysis, 4th edn. Cambridge, UK: Cambridge University Press. 